\title{
Adaptive Control of Flexible Structures with Uncertain Dynamics and Uncertain Disturbance Spectra
}

\author{
E. Dogan Sumer*and Dennis S. Bernstein ${ }^{\dagger}$ \\ University of Michigan, 1320 Beal Ave., Ann Arbor, MI 48109, USA
}

\begin{abstract}
In the present paper we revisit the active structural and acoustic vibration problem in terms of adaptive control. For active structural and acoustic vibration problem we consider retrospective cost adaptive control (RCAC). RCAC is a direct adaptive control technique, that requires minimal modeling, specifically, a limited number of Markov parameters from the control input to the performance variable. No modeling information is needed concerning the disturbance and command spectra, disturbance path, and measurement feedback path. This study is aimed at assessing in detail the level of modeling accuracy required by RCAC for disturbance rejection in lightly damped structures, with uncertain modal frequencies, damping, and mode shapes, unmodeled dynamics, uncertain disturbance spectra, sensor noise, sensor/actuator dynamics, and without the benefit of disturbance measurements, sensor/actuator passivity due to colocation and velocity sensing, in both SISO and MIMO applications.
\end{abstract}

\section{Introduction}

Active control of structural and acoustic vibration is a research problem that has enjoyed extensive attention for several decades. Applications of this technology include noise control in aircraft and buildings, active sway control in buildings and bridges, active suspension control in ground vehicles, active isolation for materials processing, and active control of flexible space structures. This paper revisits the "hopes" and "dreams" expressed in [1] on the 30th anniversary of its publication in 1982.

Some applications of active control of vibration have seen considerable success. The most successful are acoustic applications such as active headsets, where sensor and actuator hardware are relatively inexpensive, and the performance requirements can be met by modest real-time computation. These applications typically depend on direct measurements of the disturbance and rely on digital signal processing techniques and architectures that avoid feedback loops $[2,3]$. A successful area of structural vibration control includes applications where semi-active actuators are used. These devices can be controlled to modulate the damping in the structure, and thus are inherently incapable of destabilizing the structure [4-6]. Finally, active dampers for applications such as building sway are also successful due to colocated sensing and actuation, which facilitates closed-loop stability [7].

Apart from these special cases, application of structural vibration control remains a challenging problem for several reasons. First, despite considerable progress in hardware development including smart materials, vibration-control actuators tend to be expensive and require high-voltage or high-power amplifiers. Next, applications that involve unmeasured disturbances require feedback controllers and all of the difficulties

\footnotetext{
*Graduate Student, Department of Aerospace Engineering, AIAA Student Member

${ }^{\dagger}$ Professor, Department of Aerospace Engineering, AIAA Member
} 
inherent in closing a feedback loop. Although colocated rate sensing and force actuation can help guarantee stability, these configurations cannot always be achieved due to hardware constraints, for example, due to sensor dynamics and the need to measure acceleration or position. When damping augmentation cannot easily be achieved, the controller must account for the rapid and substantial phase shift that occurs at the frequency of each lightly damped mode. Uncertainty in modal frequencies, damping, and mode shapes - not to mention unmodeled dynamics and spillover as a manifestation of the Bode log-sensitivity constraint - can lead to unmodeled Nyquist encirclements and thus destabilize the closed-loop system. For applications that involve a large number of flexible modes and potential changes to the system during operation due to changes in loading and other effects, robust controllers based on small-gain analysis may not be able to roll off the loop gain while maintaining adequate phase margins [8]. Passivity technique are often the method of choice, but these entail difficulties due to sensor and actuator dynamics [9].

In the present paper we revisit the active structural and acoustic vibration problem in terms of adaptive control. Although there is no precise definition of adaptive control, it suffices to view an adaptive controller as a highly robust controller that tunes itself to the actual plant during operation and thus, unlike robust controller, avoids sacrificing performance for prior uncertainty. For active structural and acoustic vibration control we consider retrospective cost adaptive control (RCAC), which was originally developed in [16], developed in [17-21], and has subsequently been applied to diverse applications, including flight control [22].

RCAC is a direct adaptive control technique, that requires minimal modeling, specifically, a limited number of Markov parameters from the control input to the performance variable. No modeling information is needed concerning the disturbance and command spectra, disturbance path, and measurement feedback path.

The goal of this paper is to apply the latest developments in RCAC $[17-22,24]$ to the active vibration control problem. This study is aimed at assessing in detail the level of modeling accuracy required by RCAC for disturbance rejection in lightly damped structures with uncertain modal frequencies, damping, and mode shapes, unmodeled dynamics, uncertain disturbance spectra, sensor noise, sensor/actuator dynamics,

and without the benefit of disturbance measurements, and sensor/actuator passivity due to colocation and velocity sensing, in both SISO and MIMO applications.

\section{Problem Formulation}

We consider the generic structural model

$$
M \ddot{q}+C_{\mathrm{d}} \dot{q}+K q=B_{0} f+D_{w} \bar{w},
$$

where $q \in \mathbb{R}^{r}$ is a vector of generalized displacements, and $M, C_{\mathrm{d}}$, and $K$ are the mass, damping, and stiffness matrices, respectively. Throughout this paper we assume that $M$ is positive definite, and $C_{\mathrm{d}}$ and $K$ are positive semidefinite. Positive-definite and positive-semidefinite matrices are assumed to be symmetric. The control input to this system is the force $f \in \mathbb{R}^{m}$, and the disturbance force is given by $\bar{w} \in \mathbb{R}^{l_{w}}$. Measurements are given by

$$
\begin{aligned}
& \bar{y}=\left[\begin{array}{lll}
C_{0} & C_{1} & C_{2}
\end{array}\right]\left[\begin{array}{c}
q \\
\dot{q} \\
\ddot{q}
\end{array}\right]+\bar{D}_{2} \bar{v} \\
& \bar{z}=\left[\begin{array}{lll}
E_{\mathrm{p}} & E_{\mathrm{v}} & E_{\mathrm{a}}
\end{array}\right]\left[\begin{array}{c}
q \\
\dot{q} \\
\ddot{q}
\end{array}\right]+\bar{E}_{3} \bar{v}
\end{aligned}
$$


where $\bar{v}$ denotes sensor noise. The measurements $\bar{z}$ are the performance variables. We assume that $\bar{w}$ and $\bar{v}$ are uncorrelated. We can write (1), (2), (3) in state space form as

$$
\begin{aligned}
\dot{\xi}(t) & =\bar{A} \xi(t)+\bar{B} \bar{u}(t)+\bar{D}_{1} \bar{w}(t), \\
\bar{y}(t) & =\bar{C} \xi(t)+\bar{D} \bar{u}(t)+\bar{D}_{2} \bar{v}(t)+\bar{D}_{3} \bar{w}(t), \\
\bar{z}(t) & =\bar{E}_{1} \xi(t)+\bar{E}_{2} \bar{u}(t)+\bar{E}_{3} \bar{v}(t)+\bar{E}_{0} \bar{w}(t)
\end{aligned}
$$

where

$$
\begin{aligned}
\bar{A} & \triangleq\left[\begin{array}{cc}
0 & I_{r} \\
-M^{-1} K & -M^{-1} C_{\mathrm{d}}
\end{array}\right], & \bar{B} \triangleq\left[\begin{array}{c}
0_{r \times m} \\
M^{-1} B_{0}
\end{array}\right], \quad \bar{D}_{1} \triangleq\left[\begin{array}{c}
0_{r \times l_{w}} \\
M^{-1} D_{w}
\end{array}\right], \\
\bar{C} \triangleq\left[\begin{array}{ccc}
C_{0}-C_{2} M^{-1} K & C_{1}-C_{2} M^{-1} C_{\mathrm{d}}
\end{array}\right], & \bar{D} \triangleq C_{2} M^{-1} B_{0}, & \bar{D}_{3} \triangleq C_{2} M^{-1} D_{w}, \\
\bar{E}_{1} \triangleq\left[\begin{array}{cc}
E_{p}-E_{a} M^{-1} K & E_{v}-E_{a} M^{-1} C_{\mathrm{d}}
\end{array}\right], & \bar{E}_{2} \triangleq E_{a} M^{-1} B_{0}, & \bar{E}_{0} \triangleq E_{a} M^{-1} D_{w}, \\
\xi(t) & \triangleq\left[\begin{array}{c}
q(t) \\
\dot{q}(t)
\end{array}\right] \in \mathbb{R}^{2 r}, & \bar{u}(t) \triangleq f(t) .
\end{aligned}
$$

We consider four special cases of (1) when it is unforced, namely,

$$
M \ddot{q}+C_{\mathrm{d}} \dot{q}+K q=0 .
$$

These cases are distinguished by the stability of (11). For details, see [15].

In state space form, (11) can be written as

$$
\dot{\xi}=\bar{A} \xi
$$

\section{II.A. Case 1: Lyapunov-Stable Case}

The unforced structure (12) is Lyapunov stable if every eigenvalue of $\bar{A}$ lies in the closed left-half plane and is semisimple on the imaginary axis. In this case the response of (12) is bounded for all initial conditions.

Fact II.1. (12) is Lyapunov stable if and only if

$$
\operatorname{rank}\left[\begin{array}{c}
K \\
C_{\mathrm{d}}
\end{array}\right]=r .
$$

\section{II.B. Case 2: Semistable Case}

The unforced structure (12) is semistable if every eigenvalue of $\bar{A}$ lies in the open left-half plane or is zero and the zero eigenvalue (if present) is semisimple. In this case, The free response of such a structure is bounded and the state $q$ converges, but not necessarily to $q=0$.

Fact II.2. (12) is semistable if and only if $\left(M^{-1} K, C_{\mathrm{d}}\right)$ is observable.

The observability condition in Fact II.2 is known as pervasive damping.

The presence of a semisimple eigenvalue at zero signifies the presence of a damped rigid body mode. 


\section{II.C. Case 3: Asymptotically Stable Case}

The unforced structure (12) is asymptotically stable if every eigenvalue of $\bar{A}$ lies in the open left-half plane. In this case the free response of (12) converges to $q=0, \dot{q}=0$ for all initial conditions.

Fact II.3. (12) is asymptotically stable if and only if $A$ is semistable and $K$ is positive definite.

\section{II.D. Case 4: Unstable Case}

If (12) is not Lyapunov stable, then we say that (12) is unstable. The following result shows that an unstable structure must have at least one rigid body mode and that this is precisely the nature of the instability.

Fact II.4. Assume that (12) is not Lyapunov stable. Then $\bar{A}$ has a repeated zero eigenvalue that appears in a $2 \times 2$ block in the Jordan canonical form of $\bar{A}$, and no zero eigenvalue of $\bar{A}$ appears in a Jordan block of size greater than $2 \times 2$.

\section{Retrospective Cost Adaptive Control Algorithm}

\section{III.A. Discrete-time Control Problem and Useful Definitions}

Consider the MIMO discrete-time system

$$
\begin{aligned}
x(k+1) & =A x(k)+B u(k)+D_{1} w(k), \\
y(k) & =C x(k)+D_{2} w(k)+D_{3} v(k), \\
z(k) & =E_{1} x(k)+E_{0} w(k)+E_{3} v(k),
\end{aligned}
$$

where $x(k) \in \mathbb{R}^{n}, y(k) \in \mathbb{R}^{l_{y}}, z(k) \in \mathbb{R}^{l_{z}}, u(k) \in \mathbb{R}^{l_{u}}, w(k) \in \mathbb{R}^{l_{w}}, v(k) \in \mathbb{R}^{l_{v}}$, and $k \geq 0$. The system (14)-(16) can represent a sampled-data application arising from a continuous-time system with state $\xi(t)$ and sample and hold operations with sample interval $T_{\mathrm{s}}$, where $x(k), y(k), z(k), u(k), w(k)$ and $v(k)$ represent $\xi\left(k T_{\mathrm{s}}\right), \bar{y}\left(k T_{s}\right), \bar{z}\left(k T_{s}\right), \bar{u}\left(k T_{s}\right), \bar{w}\left(k T_{s}\right)$ and $\bar{v}\left(k T_{s}\right)$ respectively.

We can represent (14), (16) as the time-series model

$$
z(k)=\sum_{i=1}^{n}-\alpha_{i} z(k-i)+\sum_{i=d}^{n} \beta_{i} u(k-i)+\sum_{i=0}^{n} \gamma_{i} w(k-i)+E_{3} v(k),
$$

where $d$ is the smallest integer such that $\beta_{d}$ is not zero. The plant (14),(16) is represented by the transfer matrices

$$
\begin{aligned}
G_{z u}(\mathbf{q}) & \triangleq E_{1}(\mathbf{q} I-A)^{-1} B, \\
G_{z w}(\mathbf{q}) & \triangleq E_{1}(\mathbf{q} I-A)^{-1} D_{1}+E_{0},
\end{aligned}
$$

where $\mathbf{q}$ is the forward shift operator and, unlike the $z$-transform, (18) accounts for possibly nonzero initial conditions. For each positive integer $i$,

$$
H_{i} \triangleq E_{1} A^{i-1} B
$$

is the $i^{\text {th }}$ Markov parameter of $G_{z u}$. 
Now, consider the $n_{\mathrm{c}}^{\text {th }}$-order strictly proper LTI output feedback controller

$$
\begin{aligned}
x_{\mathrm{c}}(k+1) & =A_{\mathrm{c}} x_{\mathrm{c}}(k)+B_{\mathrm{c}} y(k), \\
u(k) & =C_{\mathrm{c}} x_{\mathrm{c}}(k),
\end{aligned}
$$

where $x_{\mathrm{c}}(k) \in \mathbb{R}^{n_{\mathrm{c}}}$. The feedback control (20)-(21) is described by $u=G_{\mathrm{c}}(\mathbf{q}) y$, where

$$
G_{\mathrm{c}}(\mathbf{q})=C_{\mathrm{c}}\left(z I-A_{\mathrm{c}}\right)^{-1} B_{\mathrm{c}} .
$$

The closed-loop system with output feedback (20)-(21) is thus given by

$$
\begin{aligned}
\tilde{x}(k+1) & =\tilde{A} \tilde{x}(k)+\tilde{D}_{1} w(k)+\tilde{F} v(k), \\
y(k) & =\tilde{C} \tilde{x}(k)+D_{2} w(k)+D_{3} v(k), \\
z(k) & =\tilde{E}_{1} \tilde{x}(k)+E_{0} w(k)+E_{3} v(k),
\end{aligned}
$$

where

$$
\begin{array}{ll}
\tilde{A} \triangleq\left[\begin{array}{cc}
A & B C_{\mathrm{c}} \\
B_{\mathrm{c}} C & A_{\mathrm{c}}
\end{array}\right], \quad \tilde{D}_{1} \triangleq\left[\begin{array}{c}
D_{1} \\
B_{\mathrm{c}} D_{2}
\end{array}\right], \quad \tilde{F} \triangleq\left[\begin{array}{c}
0_{n \times l_{v}} \\
B_{\mathrm{c}} D_{3}
\end{array}\right], \\
\tilde{C}=\left[\begin{array}{cc}
C & 0_{l_{y} \times n_{\mathrm{c}}}
\end{array}\right], & \tilde{E}_{1}=\left[\begin{array}{cc}
E_{1} & 0_{l_{z} \times n_{\mathrm{c}}}
\end{array}\right],
\end{array}
$$

and $\tilde{x}(k)=\left[\begin{array}{ll}x^{\mathrm{T}}(k) & x_{\mathrm{c}}^{\mathrm{T}}(k)\end{array}\right]^{\mathrm{T}} \in \mathbb{R}^{n+n_{\mathrm{c}}}$.

The goal is to develop an adaptive output feedback controller that minimizes the performance variable $z$ in the presence of the exogenous signal $w$ with limited modeling information about the dynamics and the exogenous signal. We assume that the measurements $y(k)$ and $z(k)$ are available for feedback.

\section{III.B. Control Law} by

We use a linear, strictly proper time-series controller of order $n_{\mathrm{c}}$ such that the control $u(k)$ is given

$$
u(k)=\theta^{\mathrm{T}}(k) \phi(k-1),
$$

where

$$
\begin{aligned}
\theta(k) & =\left[\begin{array}{llllll}
N_{1}^{\mathrm{T}}(k) & \cdots & N_{n_{\mathrm{c}}}^{\mathrm{T}}(k) & M_{1}^{\mathrm{T}}(k) & \cdots & M_{n_{\mathrm{c}}}^{\mathrm{T}}(k)
\end{array}\right]^{\mathrm{T}} \in \mathbb{R}^{n_{\mathrm{c}}\left(l_{u}+l_{y}\right) \times l_{u}}, \\
\phi(k-1) & =\left[\begin{array}{llllll}
y^{\mathrm{T}}(k-1) & \cdots & y^{\mathrm{T}}\left(k-n_{\mathrm{c}}\right) & u^{\mathrm{T}}(k-1) & \cdots & u^{\mathrm{T}}\left(k-n_{\mathrm{c}}\right)
\end{array}\right]^{\mathrm{T}} \in \mathbb{R}^{n_{\mathrm{c}}\left(l_{u}+l_{y}\right)} .
\end{aligned}
$$

The control law (26) can be reformulated as

$$
u(k)=\Phi(k-1) \Theta(k),
$$

where

$$
\begin{aligned}
\Phi(k-1) & \triangleq I_{l_{u}} \otimes \phi^{\mathrm{T}}(k-1) \in \mathbb{R}^{l_{u} \times l_{u} n_{\mathrm{c}}\left(l_{u}+l_{y}\right)}, \\
\Theta(k) & \triangleq \operatorname{vec}(\theta(k)) \in \mathbb{R}^{l_{u} n_{\mathrm{c}}\left(l_{u}+l_{y}\right)},
\end{aligned}
$$

" $\otimes$ " denotes the Kronecker product, and "vec" is the column stacking operator. 


\section{III.C. Retrospective Performance}

For positive integer $r$, we define

$$
G_{\mathrm{f}}\left(\mathbf{q}^{-1}\right) \triangleq K_{1} \mathbf{q}^{-1}+\cdots+K_{r} \mathbf{q}^{-r},
$$

which is a finite-impulse-response (FIR) transfer matrix constructed using the filter coefficients $K_{i} \in \mathbb{R}^{l_{z} \times l_{u}}$ for $1 \leq i \leq r$. Next, using $G_{\mathrm{f}}$, we define the retrospective performance variable

$$
\hat{z}(k) \triangleq z(k)+\Phi_{\mathrm{f}}(k-1) \hat{\Theta}-u_{\mathrm{f}}(k)
$$

where

$$
\begin{aligned}
\Phi_{\mathrm{f}}(k-1) & \triangleq G_{\mathrm{f}}\left(\mathbf{q}^{-1}\right) \Phi(k-1) \in \mathbb{R}^{l_{z} \times l_{u} n_{\mathrm{c}}\left(l_{u}+l_{y}\right)}, \\
u_{\mathrm{f}}(k) & \triangleq G_{\mathrm{f}}\left(\mathbf{q}^{-1}\right) u(k) \in \mathbb{R}^{l_{z}},
\end{aligned}
$$

and $\hat{\Theta} \in \mathbb{R}^{l_{u} n_{c}\left(l_{u}+l_{y}\right)}$ is an optimization variable. The retrospective performance variable (33) can be rewritten in the form

$$
\hat{z}(k) \triangleq z(k)+K_{z u}\left(\left[\begin{array}{c}
\Phi(k-2) \\
\vdots \\
\Phi(k-r-1)
\end{array}\right] \hat{\Theta}-\left[\begin{array}{c}
u(k-1) \\
\vdots \\
u(k-r)
\end{array}\right]\right) \in \mathbb{R}^{l_{z}},
$$

where $K_{z u}=\left[\begin{array}{lll}K_{1} & \cdots & K_{r}\end{array}\right] \in \mathbb{R}^{l_{z} \times r l_{u}}$.

\section{III.D. Cumulative Update Law}

For $k>0$, we define the cumulative cost function

$$
\begin{aligned}
J_{\text {cum }}(\hat{\Theta}, k) \triangleq \sum_{i=1}^{k} \lambda^{k-i}\left(\hat{z}^{\mathrm{T}}(i) \hat{z}(i)\right. & \left.+\eta(i) \hat{\Theta}^{\mathrm{T}} \Phi_{\mathrm{f}}^{\mathrm{T}}(i-1) \Phi_{\mathrm{f}}(i-1) \hat{\Theta}\right) \\
& +\lambda^{k}(\hat{\Theta}-\Theta(0))^{\mathrm{T}} P_{0}^{-1}(\hat{\Theta}-\Theta(0)),
\end{aligned}
$$

where $\lambda \in(0,1]$, and $P_{0} \in \mathbb{R}^{l_{u} n_{\mathrm{c}}\left(l_{u}+l_{y}\right) \times l_{u} n_{\mathrm{c}}\left(l_{u}+l_{y}\right)}$ is positive definite.

Fact III.1. Define $P(k) \in \mathbb{R}^{l_{u} n_{\mathrm{c}}\left(l_{u}+l_{y}\right)}$ with $P(0)=P_{0}=\beta I_{l_{u} n_{\mathrm{c}}\left(l_{u}+l_{y}\right)}$, where $\beta>0$ is a scalar. Then, for all $k>0$, the cumulative cost function (37) has the unique global minimizer

$$
\Theta(k)=\left[I-K(k) \Phi_{f}(k-1)\right] \Theta(k-1)-P(k) \Phi_{f}^{\mathrm{T}}(k-1)\left[z(k)-u_{f}(k)\right],
$$

where

$$
P(k)=\frac{1}{\lambda}\left[P(k-1)-K(k) \Phi_{f}(k-1) P(k-1)\right],
$$

and

$$
K(k) \triangleq P(k-1) \Phi_{f}^{\mathrm{T}}(k-1)\left[\frac{\lambda}{1+\eta(k)} I_{l_{z}}+\Phi_{f}(k-1) P(k-1) \Phi_{f}^{\mathrm{T}}(k-1)\right]^{-1}
$$

Proof. The result is an application of the recursive least squares theory $[13,14]$. For a complete proof, see [24]. 


\section{III.E. Phase Matching Condition}

Let $G_{z u, i j}$ denote the transfer function from the $j^{\text {th }}$ input $u_{j}$ to the $i^{\text {th }}$ output $z_{i}$, and let $G_{\mathrm{f}, i j}$ denote the $i j^{\text {th }}$ entry of $G_{\mathrm{f}}$. Then, For $\theta \in[0, \pi]$, the phase mismatch $\Delta_{i j}(\theta)$ between $G_{\mathrm{f}, i j}$ and $G_{z u, i j}$ is defined as

$$
\Delta_{i j}(\theta) \triangleq \cos ^{-1} \frac{\operatorname{Re}\left[G_{z u, i j}\left(e^{\jmath \theta}\right) \overline{G_{\mathrm{f}, i j}\left(e^{\jmath \theta}\right)}\right]}{\left|G_{z u, i j}\left(e^{\jmath \theta}\right)\right|\left|G_{\mathrm{f}, i j}\left(e^{\jmath \theta}\right)\right|} \in[0,180] .
$$

Note that $\Delta_{i j}(\theta)$ represents the angle between $G_{z u, i j}\left(e^{\jmath \theta}\right)$ and $G_{\mathrm{f}, i j}\left(e^{\jmath \theta}\right)$ in the complex plane. The role of phase mismatch in closed-loop performance of RCAC for SISO plants is investigated in [21]. Furthermore, frequency domain methods are presented for approximating IIR plants with FIR transfer functions in [23]

\section{Numerical Examples}

We now apply RCAC to structural models. We consider disturbance rejection problems for both SISO and MIMO plants. In all cases, the adaptive controller gain matrix is initialized to be zero, that is, $\Theta(0)=0$, and the forgetting factor $\lambda=1$ in all examples.

Each example is constructed using the multiple degrees-of-freedom (MDOF) lumped parameter structure shown in Figure 1, and the output measurement is sampled with zero-order hold. The equations of motion for this system can be written in the form (1) with $M=\operatorname{diag}\left(m_{1}, \ldots, m_{r}\right)$,

$$
\begin{aligned}
C_{d} & =\left[\begin{array}{cccccc}
c_{1}+c_{2} & -c_{2} & 0 & \ldots & & 0 \\
-c_{2} & c_{2}+c_{3} & -c_{3} & 0 & \ldots & 0 \\
& \ddots & \ddots & \ddots & \ddots & \vdots \\
0 & \ldots & -c_{r-1} & c_{r-1}+c_{r} & -c_{r} & 0 \\
0 & \ldots & & 0 & -c_{r} & c_{r}+c_{r+1}
\end{array}\right] \\
K= & {\left[\begin{array}{cccccc}
k_{1}+k_{2} & -k_{2} & 0 & \ldots & & 0 \\
-k_{2} & k_{2}+k_{3} & -k_{3} & 0 & \ldots & 0 \\
& \ddots & \ddots & \ddots & \ddots & \vdots \\
0 & \ldots & -k_{r-1} & k_{r-1}+k_{r} & -k_{r} & 0 \\
0 & \ldots & & 0 & -k_{r} & k_{r}+k_{r+1}
\end{array}\right] . }
\end{aligned}
$$

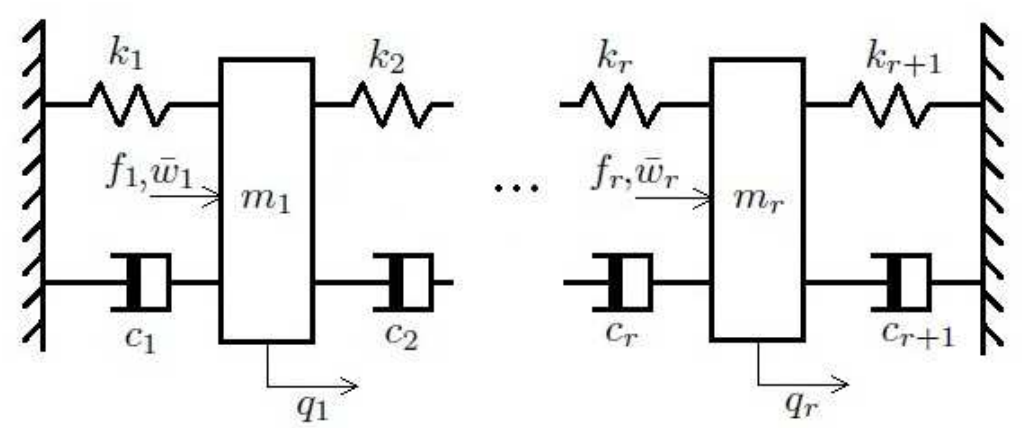

Figure 1. An r-mass lumped parameter structure. 


\section{IV.A. SISO Examples}

In this section, we apply RCAC to SISO structures. The simulation results corresponding to the examples in this section are given in Appendix A.

Example IV.1 (Adaptive control of a 2DOF asymptotically stable lumped parameter structure). Consider a two-mass lumped parameter structure with the masses $m_{1}=1, m_{2}=1$; the spring constants $k_{1}=5 \mathrm{~kg} / \mathrm{sec}^{2}, k_{2}=0 \mathrm{~kg} / \mathrm{sec}^{2}, k_{3}=2 \mathrm{~kg} / \mathrm{sec}^{2}$; and the damping coefficients $c_{1}=2 \mathrm{~kg} / \mathrm{sec}, c_{2}=1 \mathrm{~kg} / \mathrm{sec}$, and $c_{3}=0 \mathrm{~kg} / \mathrm{sec}$. With these parameters, every eigenvalue of $\bar{A}$ lies in the open left-half plane, thus the structure is asymptotically stable. The continuous-time plant $T_{z u}(s)=\bar{E}_{1}(s I-\bar{A})^{-1} \bar{B}$ is sampled at $4 \mathrm{~Hz}$ so that $T_{s}=0.25 \mathrm{sec} /$ sample. The sampled-data system $G_{z u}(\mathbf{q})$ has the sampling zeros -0.211 and -2.8758 , one of which is nonminimum-phase.

The control objective is to keep $q_{2}$ near zero in the presence of the disturbance forces $\bar{w}_{1}$ and $\bar{w}_{2}$, using the control force $f_{1}$. Therefore, we consider a SISO disturbance rejection problem with $z=q_{2}$, $B_{0}=\left[\begin{array}{ll}1 & 0\end{array}\right]^{\mathrm{T}}, D_{w}=I_{2}$. We assume that $q_{2}$ is the only measurement, therefore, $y=z$. Furthermore, we assume that the measurements are noise-free.

We first consider an unknown sinusoidal disturbance $\bar{w}_{2}(t)$ with frequency $\omega_{2}=\frac{1}{7} \mathrm{~Hz}$, that is, $w(k)=$ $\left[\begin{array}{ll}0 & 100 \sin \Theta_{2} k\end{array}\right]^{\mathrm{T}} \mathrm{N}$, where $\Theta_{2}=2 \pi \omega_{2} T_{s}=2 \pi / 28 \mathrm{rad} /$ sample. The open-loop system is given the initial conditions $q(0)=\left[\begin{array}{ll}4 & -1\end{array}\right]^{\mathrm{T}} \mathrm{m}$, and $\dot{q}(0)=\left[\begin{array}{ll}-2 & -0.5\end{array}\right]^{\mathrm{T}} \mathrm{m} /$ sec. The plant is simulated in open-loop for 25 seconds, and at $t=25 \mathrm{sec}, \mathrm{RCAC}$ is turned on with tuning parameters $n_{\mathrm{c}}=10, \eta_{0}=0.5, P_{0}=10 I$, $p_{c}=1$, and $K_{z u}=H_{1}=0.002$. The performance converges to zero, the asymptotic closed-loop system is stable, and RCAC converges to an internal model controller with high-gain at the disturbance frequency $\Theta_{2}$ as shown in Figure 13.

We now consider the unknown sinusoidal disturbances $\bar{w}_{1}(t)$ and $\bar{w}_{2}(t)$ with frequencies $\omega_{1}=0.5 \mathrm{~Hz}$ and $\omega_{2}=\frac{2}{9} \mathrm{~Hz}$, that is, $w(k)=\left[\begin{array}{ll}w_{1}(k) & w_{2}(k)\end{array}\right]^{\mathrm{T}}=\left[\begin{array}{ll}100 \sin \Theta_{1} k & 10 \sin \Theta_{2} k\end{array}\right]^{\mathrm{T}} \mathrm{N}$, where $\Theta_{1}=2 \pi / 8$ $\mathrm{rad} /$ sample and $\Theta_{2}=2 \pi / 18 \mathrm{rad} /$ sample. We choose

$$
K_{z u}=\left[\begin{array}{lll}
K_{1} & \cdots & K_{7}
\end{array}\right]=10^{-3}\left[\begin{array}{llllllll}
-2.1 & 0.3 & 1.4 & 1.6 & 1.2 & 0.2 & -0.7 & -1.8
\end{array}\right],
$$

so that $\Delta(\theta)<90$ for all $\theta \in[0, \pi] \mathrm{rad} /$ sample. Note that the NMP sampling zero -2.8758 is not a zero of $G_{\mathrm{f}}$. The open-loop system is given the same initial conditions as above. The plant is simulated in open-loop for 100 seconds, and at $t=100 \mathrm{sec}, \mathrm{RCAC}$ is turned on with tuning parameters $n_{\mathrm{c}}=15, \eta_{0}=0.1, P_{0}=0.1 I$, and $p_{c}=5$. The closed-loop response is shown in Figure 14. After convergence, the disturbance frequencies $\pi / 9 \mathrm{rad} /$ sample and $\pi / 4 \mathrm{rad} /$ sample are attenuated as shown in Figure 2.
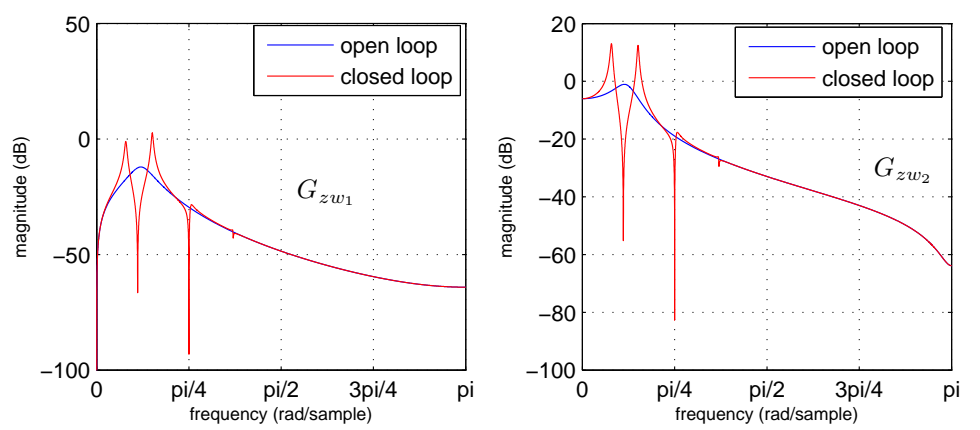

Figure 2. Example IV.1: 2DOF asymptotically stable structure with nonminimum-phase sampling zeros, two-tone disturbance rejection problem. The bode plots show the attenuation at the disturbance frequencies $\pi / 9$ and $\pi / 4$ $\mathrm{rad} / \mathrm{sample}$ after controller convergence.

Example IV.2 (Adaptive control of a 3DOF asymptotically stable lumped parameter structure). Consider a 3 DOF lumped parameter structure with the masses $m_{1}=1.1 \mathrm{~kg}, m_{2}=0.7 \mathrm{~kg}, m_{3}=1 \mathrm{~kg}$; the 
spring constants $k_{1}=0.5 \mathrm{~kg} / \mathrm{sec}^{2}, k_{2}=0.01 \mathrm{~kg} / \mathrm{sec}^{2}, k_{3}=8 \mathrm{~kg} / \mathrm{sec}^{2}, k_{4}=4 \mathrm{~kg} / \mathrm{sec}^{2} ;$ and the damping coefficients $c_{1}=1.5 \mathrm{~kg} / \mathrm{sec}, c_{2}=0.5 \mathrm{~kg} / \mathrm{sec}, c_{3}=0.8 \mathrm{~kg} / \mathrm{sec}$, and $c_{4}=0 \mathrm{~kg} / \mathrm{sec}$. With these parameters, every eigenvalue of $\bar{A}$ lies in the open left-half plane, and thus the structure is asymptotically stable. The continuous-time plant $T_{z u}(s)$ is sampled at $5 \mathrm{~Hz}$ so that $T_{s}=0.2 \mathrm{sec} / \mathrm{sample}$. The sampled-data system $G_{z u}(z)$ has the minimum-phase sampling zero -0.8779 .

The control objective is to keep $q_{2}$ near zero in the presence of the disturbance forces $\bar{w}_{1}(t), \bar{w}_{2}(t)$ and $\bar{w}_{3}(t)$, using the control force $f_{2}$. Therefore, we consider a SISO disturbance rejection problem with $z=q_{2}$, $B_{0}=\left[\begin{array}{lll}0 & 1 & 0\end{array}\right]^{\mathrm{T}}, D_{w}=I_{3}$. We assume that $q_{2}$ is the only measurement, therefore, $y=z$. Furthermore, we assume that the measurements are noise-free.

We consider unknown sinusoidal disturbances $\bar{w}_{1}, \bar{w}_{2}$ and $\bar{w}_{3}$ with frequencies $\omega_{1}=\frac{5}{14} \mathrm{~Hz}, \omega_{2}=\frac{5}{36} \mathrm{~Hz}$, $\omega_{3}=\frac{5}{3} \mathrm{~Hz}$, that is, $w=\left[\begin{array}{lll}w_{1} & w_{2} & w_{3}\end{array}\right]^{\mathrm{T}}=\left[\begin{array}{lll}10 \sin \Theta_{1} k & 2 \sin \Theta_{2} k & 15 \sin \Theta_{3} k\end{array}\right]^{\mathrm{T}} \mathrm{N}$, where $\Theta_{1}=2 \pi / 14$ $\mathrm{rad} /$ sample, $\Theta_{2}=2 \pi / 36 \mathrm{rad} /$ sample, and $\Theta_{3}=2 \pi / 3 \mathrm{rad} /$ sample. The open-loop system is given the initial conditions $q(0)=\left[\begin{array}{lll}5 & -1.5 & -3\end{array}\right]^{\mathrm{T}} \mathrm{m}$, and $\dot{q}=\left[\begin{array}{lll}-0.5 & -7 & -3.5\end{array}\right]^{\mathrm{T}} \mathrm{m} / \mathrm{sec}$. The plant is simulated in open-loop for 15 seconds, and at $t=15 \mathrm{sec}, \mathrm{RCAC}$ is turned on with tuning parameters $n_{\mathrm{c}}=15, \eta_{0}=0$, $P_{0}=10^{10} I$, and $K_{z u}=H_{1}=0.0246$. The closed-loop response is shown in Figure 15. After convergence, the disturbance frequencies $\pi / 7 \mathrm{rad} /$ sample, $\pi / 18 \mathrm{rad} /$ sample and $2 \pi / 3 \mathrm{rad} /$ sample are attenuated as shown in Figure 2.
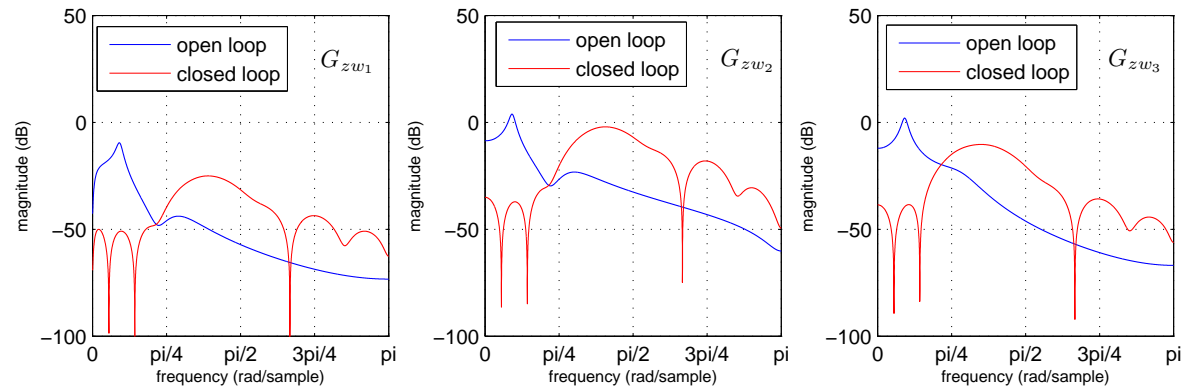

Figure 3. Example IV.2: 3DOF asymptotically stable structure, three-tone disturbance rejection problem. The bode plots show the attenuation at the disturbance frequencies $\pi / 7, \pi / 18$ and $2 \pi / 3 \mathrm{rad} / \mathrm{sample}$ after controller convergence.

Example IV.3 (Broadband disturbance rejection in a 3DOF asymptotically stable structure). Consider a 3DOF lumped parameter structure with the masses $m_{1}=1.8 \mathrm{~kg}, m_{2}=0.7 \mathrm{~kg}, m_{3}=1.11 \mathrm{~kg}$; the spring constants $k_{1}=7.5 \mathrm{~kg} / \mathrm{sec}^{2}, k_{2}=4.9 \mathrm{~kg} / \mathrm{sec}^{2}, k_{3}=7 \mathrm{~kg} / \mathrm{sec}^{2}, k_{4}=7.5 \mathrm{~kg} / \mathrm{sec}^{2}$; and the damping coefficients $c_{1}=0.8 \mathrm{~kg} / \mathrm{sec}, c_{2}=0.6 \mathrm{~kg} / \mathrm{sec}, c_{3}=0.2 \mathrm{~kg} / \mathrm{sec}$, and $c_{4}=0.45 \mathrm{~kg} / \mathrm{sec}$. With these parameters, every eigenvalue of $\bar{A}$ lies in the open left-half plane, and thus the structure is asymptotically stable. The continuous-time plant $T_{z u}(s)$ is sampled at $6.667 \mathrm{~Hz}$ so that $T_{s}=0.15 \mathrm{sec} / \mathrm{sample}$. The sampled-data system $G_{z u}(z)$ has the minimum-phase sampling zero -0.9424 .

The control objective is to keep $q_{2}$ near zero in the presence of the bandlimited white disturbances $w_{1}(k), w_{2}(k)$ and $w_{3}(k)$, using the control force $f_{2}$. The disturbances are assumed to be uncorrelated with each other, have the standard deviations $\sigma_{w_{1}}=157.29 \mathrm{~N}, \sigma_{w_{2}}=99.81 \mathrm{~N}, \sigma_{w_{3}}=153.97 \mathrm{~N}$, and the correlation time of each bandlimited disturbance is equal to $0.2 T_{s}=0.03 \mathrm{sec}$. We assume that the measurements are noise-free, and $q_{2}$ is the only measurement, therefore, $y=z=q_{2}$. The open-loop system is given the initial conditions $q(0)=\left[\begin{array}{lll}-0.05 & 0.3 & 0.15\end{array}\right]^{\mathrm{T}} \mathrm{m}$, and $\dot{q}(0)=\left[\begin{array}{ccc}-0.4 & 0.4 & 0.25\end{array}\right]^{\mathrm{T}} \mathrm{m} / \mathrm{sec}$. The plant is simulated in open-loop for 300 seconds, and at $t=300 \mathrm{sec}$, RCAC is turned on with tuning parameters $n_{\mathrm{c}}=6, \eta_{0}=0, P_{0}=100 I$, and $K_{z u}=H_{1}=0.0147$. The performance variable is brought below open-loop level as shown in Figure 16. After convergence, the lightly-damped mode is suppressed as shown in Figure 4.

Example IV.4 (Adaptive control of a 2DOF semistable lumped parameter structure with sensor noise). Consider a 2DOF lumped parameter structure with the masses $m_{1}=2 \mathrm{~kg}, m_{2}=0.6 \mathrm{~kg}$; the spring 

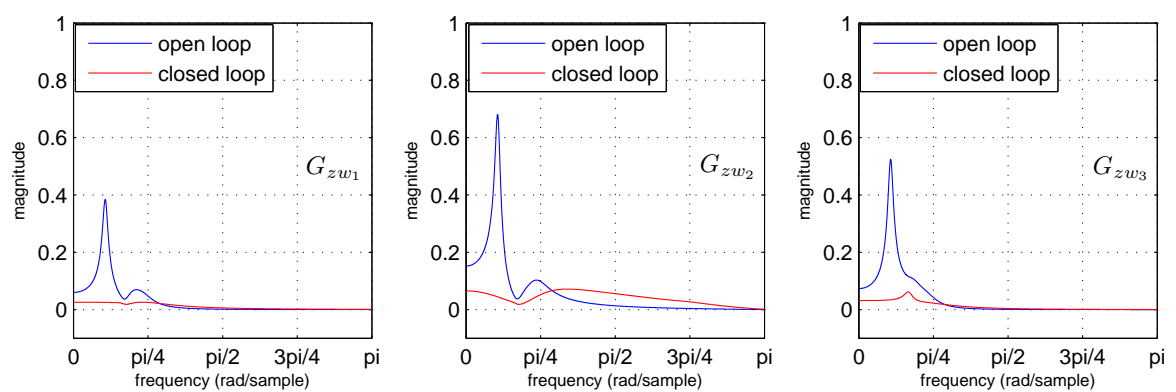

Figure 4. Example IV.3: 3DOF asymptotically stable structure, broadband disturbance rejection problem. The bode plots show the suppression of the lightly-damped mode in each path, after controller convergence.

constants $k_{1}=10 \mathrm{~kg} / \mathrm{sec}^{2}, k_{2}=0 \mathrm{~kg} / \mathrm{sec}^{2}, k_{3}=0 \mathrm{~kg} / \mathrm{sec}^{2}$; and the damping coefficients $c_{1}=0.5 \mathrm{~kg} / \mathrm{sec}$, $c_{2}=0.4 \mathrm{~kg} / \mathrm{sec}$, and $c_{3}=0.4 \mathrm{~kg} / \mathrm{sec}$. With these parameters, the structure has a damped rigid-body mode, which results in $\bar{A}$ having an eigenvalue at the origin. The remaining eigenvalues lie in the open left-half plane, therefore, the structure is semistable. The continuous-time plant $T_{z u}(s)$ is sampled at $2 \mathrm{~Hz}$ so that $T_{s}=0.5 \mathrm{sec} /$ sample. The sampled-data system $G_{z u}(z)$ has one minimum-phase sampling zero.

The control objective is to keep $q_{2}$ near zero in the presence of the disturbance forces $\bar{w}_{1}$ and $\bar{w}_{2}$, using the control force $f_{2}$. We first assume that the measurements are noise-free, and $q_{2}$ is the only measurement, therefore, $y=z=q_{2}$. We consider the unknown disturbance vector $w(k)=\left[\begin{array}{ll}w_{1}(k) & w_{2}(k)\end{array}\right]^{\mathrm{T}}=$ $\left[100 \sin 2 \pi \omega_{1} T_{s} k \quad 1+10 \sin 2 \pi \omega_{2} T_{s} k\right]^{\mathrm{T}}$, where $\omega_{1}=\frac{2}{3} \mathrm{~Hz}$ and $\omega_{2}=\frac{2}{5} \mathrm{~Hz}$. Note that $w_{2}$ has a nonzero DC component that excites the damped rigid-body mode of the structure. The open-loop system is given the initial conditions $q(0)=\left[\begin{array}{ll}2.5 & -3.5\end{array}\right]^{\mathrm{T}} \mathrm{m}$, and $\dot{q}=\left[\begin{array}{ll}-2.5 & -5\end{array}\right]^{\mathrm{T}} \mathrm{m} / \mathrm{sec}$. The plant is simulated in open-loop for 10 seconds, and at $t=10 \mathrm{sec}, \mathrm{RCAC}$ is turned on with tuning parameters $n_{\mathrm{c}}=10, \eta_{0}=0$, $P_{0}=I$, and $K_{z u}=H_{1}=0.1692$. The closed-loop response is shown in Figure 17. After convergence, the disturbance frequencies $2 \pi / 3 \mathrm{rad} /$ sample, $2 \pi / 5 \mathrm{rad} /$ sample and the $\mathrm{DC}$ component are attenuated as shown in Figure 5.

We now add measurement noise to $y$ and $z$, therefore, $y(k)=z(k)=q_{2}\left(k T_{s}\right)+v(k)$, where $v$ is a zero-mean gaussian white-noise with standard deviation $\sigma_{v}=0.44 \mathrm{~m}$. We consider the same disturbance forces $w_{1}$ and $w_{2}$ with the same initial conditions $q(0)$ and $\dot{q}(0)$. The plant is simulated in open-loop for 10 seconds, and at $t=10 \mathrm{sec}, \mathrm{RCAC}$ is turned on with tuning parameters $n_{\mathrm{c}}=10, \eta_{0}=0, P_{0}=I$, and $K_{z u}=H_{1}=0.1692$. The closed-loop response is shown in Figure 18. After convergence, the disturbance frequencies $2 \pi / 3 \mathrm{rad} / \mathrm{sample}, 2 \pi / 5 \mathrm{rad} /$ sample and the $\mathrm{DC}$ component are attenuated as shown in Figure 5 .
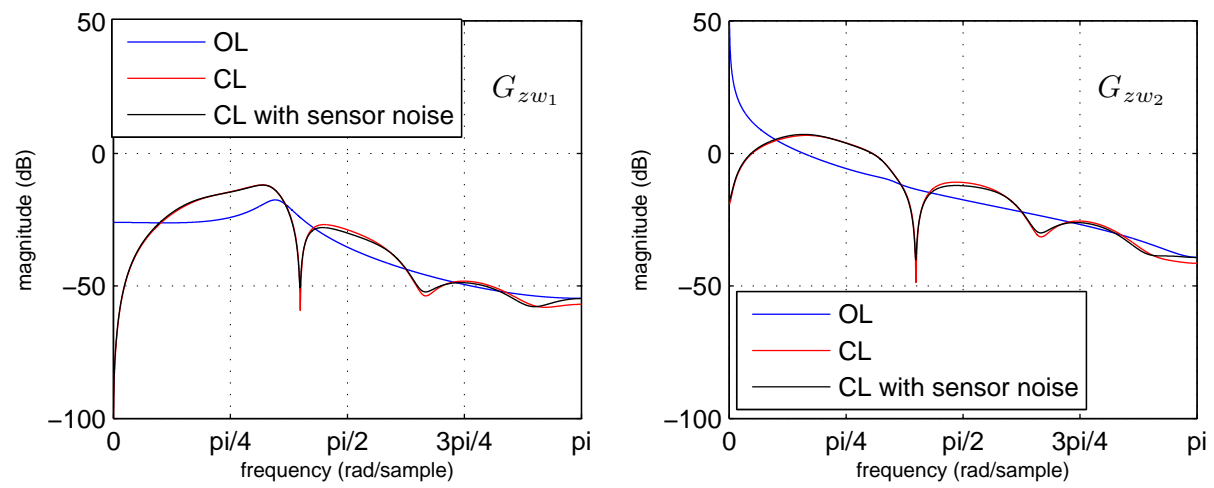

Figure 5. Example IV.4: 2DOF semistable structure. The bode plots show the attenuation at the disturbance frequencies $2 \pi / 3,2 \pi / 5 \mathrm{rad} / \mathrm{sample}$ as well as the DC component after controller convergence. Sensor noise does not hinder attenuation of the disturbance frequencies. 
Example IV.5 (3DOF asymptotically stable lumped parameter structure with measurement noise). Consider a 3 DOF lumped parameter structure with the masses $m_{1}=5.1 \mathrm{~kg}, m_{2}=3.9 \mathrm{~kg}, m_{3}=6.4$ $\mathrm{kg}$; the spring constants $k_{1}=11 \mathrm{~kg} / \mathrm{sec}^{2}, k_{2}=6 \mathrm{~kg} / \mathrm{sec}^{2}, k_{3}=7 \mathrm{~kg} / \mathrm{sec}^{2}, k_{4}=11 \mathrm{~kg} / \mathrm{sec}$; and the damping coefficients $c_{1}=3 \mathrm{~kg} / \mathrm{sec}, c_{2}=2.9 \mathrm{~kg} / \mathrm{sec}, c_{3}=2.92 \mathrm{~kg} / \mathrm{sec}$, and $c_{4}=2.99 \mathrm{~kg} / \mathrm{sec}$. With these parameters, every eigenvalue of $\bar{A}$ lies in the open left-half plane, therefore, the structure is asymptotically stable. Numerical values of $M, C_{\mathrm{d}}$ and $K$ are

$$
M=\left[\begin{array}{ccc}
5.1 & 0 & 0 \\
0 & 3.9 & 0 \\
0 & 0 & 6.4
\end{array}\right], C_{\mathrm{d}}=\left[\begin{array}{ccc}
5.9 & -2.9 & 0 \\
-2.9 & 5.82 & -2.92 \\
0 & -2.92 & 5.91
\end{array}\right], K=\left[\begin{array}{ccc}
17 & -6 & 0 \\
-6 & 13 & -7 \\
0 & -7 & 18
\end{array}\right]
$$

The continuous-time plant $T_{z u}(s)$ is sampled at $2.5 \mathrm{~Hz}$ so that $T_{s}=0.4 \mathrm{sec} / \mathrm{sample}$.

The control objective is to keep $q_{2}$ near zero in the presence of the disturbance forces $\bar{w}_{1}$, $\bar{w}_{2}$ and $\bar{w}_{3}$ using the control force $f_{2}$. Throughout the example, we assume that the measurements $y$ and $z$ are corrupted by a zero-mean gaussian white noise $v(k)$ with standard deviation $\sigma_{v}=0.24 \mathrm{~m}$, so that $y(k)=z(k)=q_{2}\left(k T_{s}\right)+v(k)$. We consider the unknown sinusoidal disturbances $\bar{w}_{1}, \bar{w}_{2}$ and $\bar{w}_{3}$ with frequencies $\omega_{1}=0.625 \mathrm{~Hz}, \omega_{2}=0.1786 \mathrm{~Hz}, \omega_{3}=0.4167 \mathrm{~Hz}$, that is, $w(k)=\left[\begin{array}{lll}w_{1}(k) & w_{2}(k) & w_{3}(k)\end{array}\right]^{\mathrm{T}}=$ $\left[\begin{array}{lll}80 \sin \Theta_{1} k & 15 \sin \Theta_{2} k & 70 \sin \Theta_{3} k\end{array}\right]^{\mathrm{T}} \mathrm{N}$, where $\Theta_{1}=\pi / 2 \mathrm{rad} /$ sample, $\Theta_{2}=\pi / 7 \mathrm{rad} / \mathrm{sample}$, and $\Theta_{3}=$ $\pi / 3 \mathrm{rad} /$ sample. The open-loop system is given the initial conditions $q(0)=\left[\begin{array}{lll}-0.1 & -0.6 & -0.75\end{array}\right]^{\mathrm{T}} \mathrm{m}$, and $\dot{q}=\left[\begin{array}{ccc}0.4 & 0.1 & 0\end{array}\right]^{\mathrm{T}} \mathrm{m} / \mathrm{sec}$. The plant is simulated in open-loop for 40 seconds, and at $t=40 \mathrm{sec}$, RCAC is turned on with tuning parameters $n_{\mathrm{c}}=15, \eta_{0}=0, P_{0}=10^{3} I$, and $K_{z u}=H_{1}=0.0165$. The closed-loop response is shown in Figure 19. After convergence, the disturbance frequencies $\pi / 2 \mathrm{rad} / \mathrm{sample}$, $\pi / 3 \mathrm{rad} /$ sample and $\pi / 7$ are attenuated as shown in Figure 6.
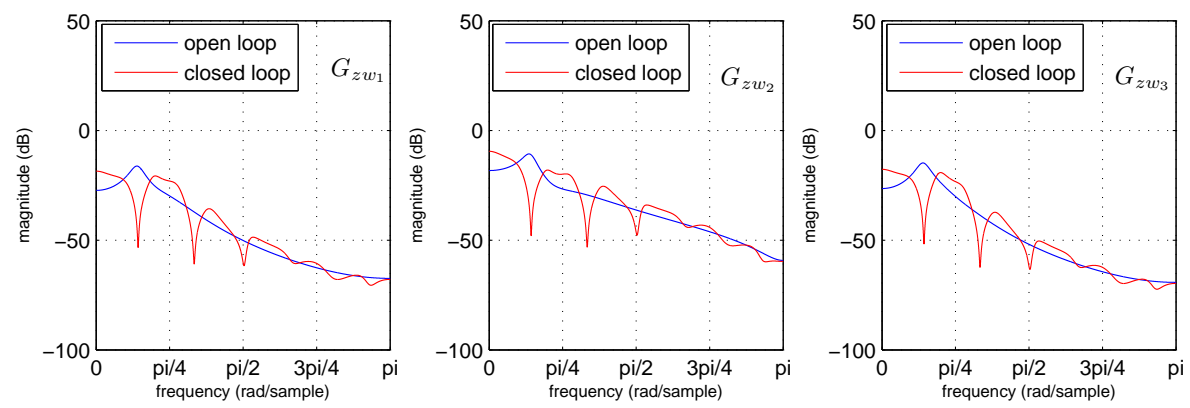

Figure 6. Example IV.5: 3DOF asymptotically stable structure. The bode plots show the attenuation at the disturbance frequencies $\pi / 2, \pi / 3$ and $\pi / 7 \mathrm{rad} /$ sample after controller convergence.

Example IV.6 (3DOF asymptotically stable lumped parameter structure with uncertain dynamics and measurement noise). We now consider the same structure as in Example IV.5, but introduce the random uncertainties $S_{M}, S_{C_{\mathrm{d}}}, S_{K} \in \mathbb{R}^{3 \times 3}$ so that

$$
\tilde{M}=M+S_{M} S_{M}^{\mathrm{T}}, \quad \tilde{C}_{\mathrm{d}}=C_{\mathrm{d}}+S_{C_{\mathrm{d}}} S_{C_{\mathrm{d}}}^{\mathrm{T}} \quad \tilde{K}=[K]+S_{K} S_{K}^{\mathrm{T}},
$$

where $M, C, K$ have the same values as introduced in (43). The uncertainties are normalized so that $\left\|S_{M} S_{M}^{\mathrm{T}}\right\|_{2}=\|M\|_{2},\left\|S_{C_{\mathrm{d}}} S_{C_{\mathrm{d}}}^{\mathrm{T}}\right\|_{2}=\left\|C_{\mathrm{d}}\right\|_{2}$, and $\left\|S_{K} S_{K}^{\mathrm{T}}\right\|_{2}=\|K\|_{2}$. We therefore consider the uncertain structure (1) where the mass, damping and stiffness matrices are $\tilde{M}, \tilde{C_{\mathrm{d}}}$, and $\tilde{K}$ respectively. The numerical values of $\tilde{M}, \tilde{C_{\mathrm{d}}}$ and $\tilde{K}$ are

$\tilde{M}=\left[\begin{array}{ccc}10.047 & 0.191 & -2.431 \\ 0.191 & 4.724 & -0.605 \\ -2.431 & -0.605 & 8.579\end{array}\right], \tilde{C_{\mathrm{d}}}=\left[\begin{array}{ccc}11.305 & -1.270 & -4.402 \\ -1.270 & 7.247 & -4.493 \\ -4.402 & -4.493 & 10.413\end{array}\right], \tilde{K}=\left[\begin{array}{ccc}24.795 & -15.775 & 2.379 \\ -15.775 & 31.308 & -9.982 \\ 2.379 & -9.982 & 21.086\end{array}\right]$. 
The additive uncertainties change the frequency response of $T_{z u}(s)$ as shown in Figure 7.
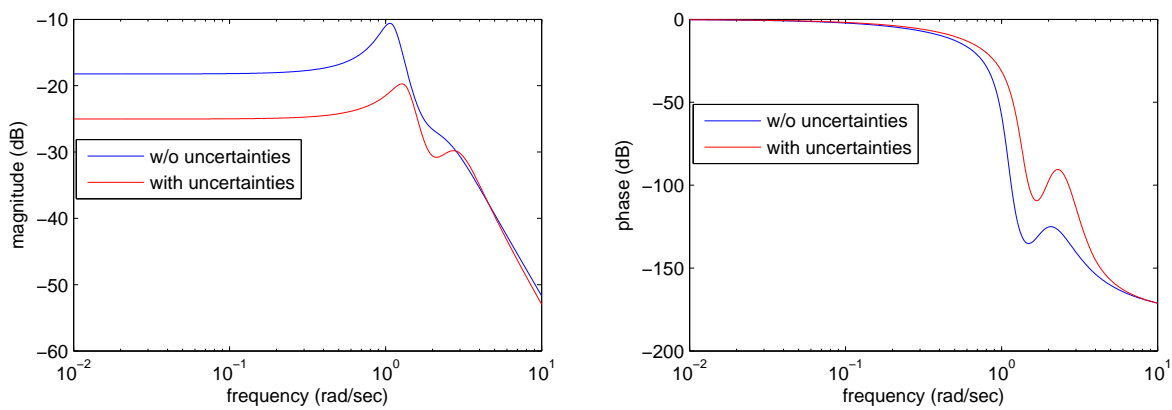

Figure 7. Example IV.6: 3DOF asymptotically stable structure with uncertain dynamics. Blue represents the frequency response of the original plant $T_{z u}(s)$ given in Example IV.5, and red illustrates the frequency response of the plant with the unknown additive uncertainties $S_{M}, S_{C_{d}}$ and $S_{K}$.

We consider the same disturbances $\bar{w}_{1}, \bar{w}_{2}$ and $\bar{w}_{3}$ with the same initial conditions $q(0), \dot{q}(0)$ as in Example IV.5. We use the same $K_{z u}=K_{1}=0.0165$, which is not equal to the first Markov parameter $H_{1}=0.0133$ of the uncertain system. The plant is simulated in open-loop for 40 seconds, and at $t=40 \mathrm{sec}$, RCAC is turned on with tuning parameters $n_{\mathrm{c}}=15, \eta_{0}=0$, and $P_{0}=10^{3} I$. The closed-loop response is shown in Figure 20. After convergence, the disturbance frequencies $\pi / 2 \mathrm{rad} / \mathrm{sample}, \pi / 3 \mathrm{rad} / \mathrm{sample}$ and $\pi / 7$ are attenuated as shown in Figure 8.
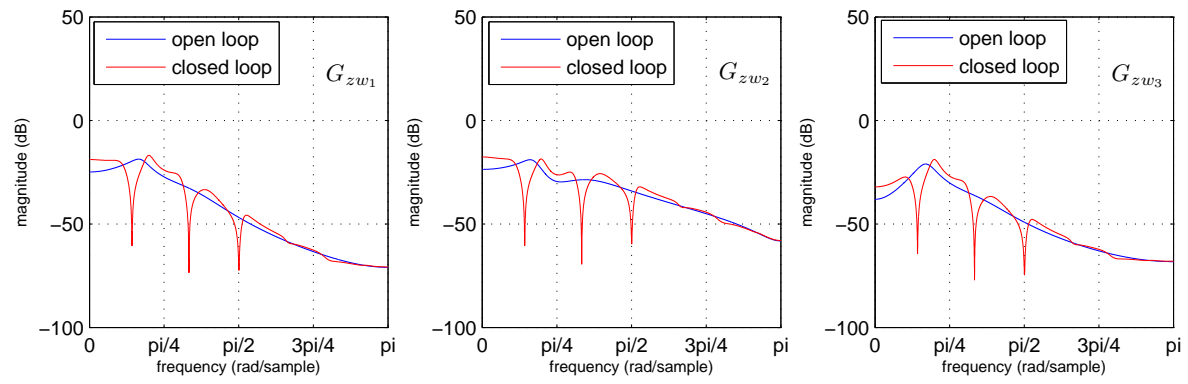

Figure 8. Example IV.6: 3DOF asymptotically stable structure with unknown additive uncertainties. The bode plots show the attenuation at the disturbance frequencies $\pi / 2, \pi / 3$ and $\pi / 7 \mathrm{rad} /$ sample after controller convergence.

Example IV.7 (3DOF asymptotically stable lumped parameter structure with uncertain dynamics and measurement noise). Consider a 3DOF lumped parameter structure with the masses $m_{1}=4.6 \mathrm{~kg}$, $m_{2}=4.7 \mathrm{~kg}, m_{3}=5.2 \mathrm{~kg}$; the spring constants $k_{1}=9.9 \mathrm{~kg} / \mathrm{sec}^{2}, k_{2}=5.8 \mathrm{~kg} / \mathrm{sec}^{2}, k_{3}=8 \mathrm{~kg} / \mathrm{sec}^{2}, k_{4}=8.5$ $\mathrm{kg} / \mathrm{sec}$; and the damping coefficients $c_{1}=2.8 \mathrm{~kg} / \mathrm{sec}, c_{2}=2.7 \mathrm{~kg} / \mathrm{sec}, c_{3}=2.42 \mathrm{~kg} / \mathrm{sec}$, and $c_{4}=2.65$ $\mathrm{kg} / \mathrm{sec}$. With these parameters, every eigenvalue of $\bar{A}$ lies in the open left-half plane, therefore, the structure is asymptotically stable. The continuous-time plant $T_{z u}(s)$ is sampled at $1 \mathrm{~Hz}$ so that $T_{s}=1 \mathrm{sec} / \mathrm{sample}$. The sampled-data system $G_{z u}(z)$ has two sampling zeros, one of which is nonminimum-phase.

In this example, we assume that the structure parameters, including the Markov parameters, are completely unknown, that is, no prior modeling information is available. Therefore, we first apply an off-line frequency-domain identification to construct $K_{z u}$. In particular, with the unknown nonzero initial conditions $q(0)=\left[\begin{array}{lll}0.04 & 0.1 & -0.02\end{array}\right]^{\mathrm{T}} \mathrm{m}$ and $\dot{q}(0)=\left[\begin{array}{lll}0.02 & -0.03 & 0.01\end{array}\right]^{\mathrm{T}} \mathrm{m} / \mathrm{sec}$, we excite the uncertain plant with a white noise sequence and collect output measurements for 1500 time steps. We then take the ratio of the fast fourier transforms of the output and input signals to obtain frequency response estimates $\hat{G}_{z u}\left(e^{\jmath \theta}\right)$ of $G_{z u}$ in 750 equally spaced points in $\theta \in[0, \pi] \mathrm{rad} /$ sample. The bode plot of the estimated frequency response is shown in Figure 9. Next, using the frequency response estimates, we apply a constrained linear least squares method to fit $\hat{G}_{z u}\left(e^{\jmath \theta}\right)$ with an FIR plant using a uniform phase mismatch bound $\Delta(\theta) \leq 80$ deg. The resulting FIR fit is

$$
G_{\mathrm{f}}\left(q^{-1}\right)=0.041 q^{-1}+0.0709 q^{-2}
$$


hence, we choose $K_{z u}=\left[\begin{array}{ll}0.041 & 0.0709\end{array}\right]$. Note that the entries of $K_{z u}$ are not the Markov parameters $H_{1}=0.0155$ and $H_{2}=0.0578$ of $G_{z u}$.
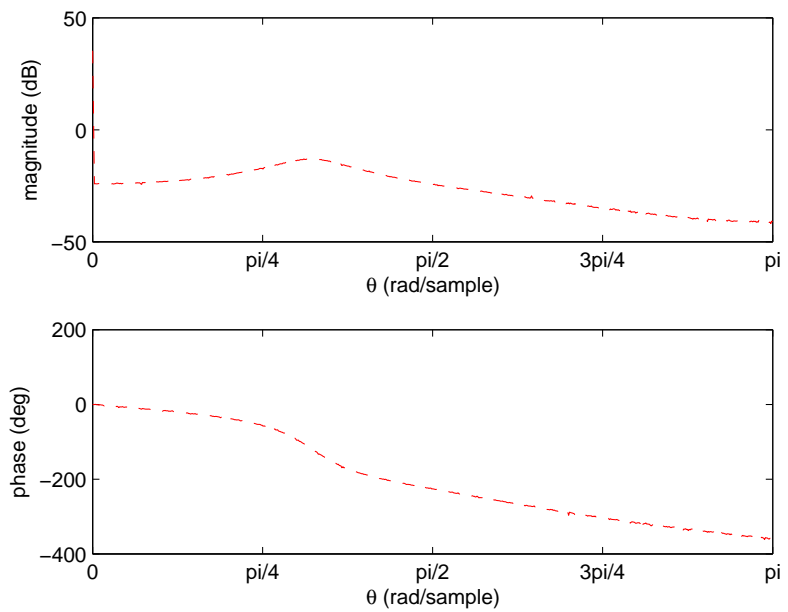

Figure 9. Example IV.7: Frequency response estimate of $G_{z u}$, obtained through frequency domain system identification using a gaussian white noise sequence.

The control objective is to keep $q_{2}$ near zero in the presence of the disturbance forces $\bar{w}_{1}, \bar{w}_{2}$ and $\bar{w}_{3}$ using the control force $f_{3}$. Furthermore, we assume that the measurements $y$ and $z$ are corrupted by a zeromean gaussian white noise $v(k)$ with standard deviation $\sigma_{v}=0.32 \mathrm{~m}$, so that $y(k)=z(k)=q_{2}\left(k T_{s}\right)+v(k)$. We consider the unknown sinusoidal disturbances $\bar{w}_{1}, \bar{w}_{2}$ and $\bar{w}_{3}$ with frequencies $\omega_{1}=0.1429 \mathrm{~Hz}, \omega_{2}=\frac{1}{3}$ $\mathrm{Hz}, \omega_{3}=0.0588 \mathrm{~Hz}$, that is, $w(k)=\left[\begin{array}{lll}w_{1}(k) & w_{2}(k) & w_{3}(k)\end{array}\right]^{\mathrm{T}}=\left[\begin{array}{ccc}75 \sin \Theta_{1} k & 30 \sin \Theta_{2} k & 70 \sin \Theta_{3} k\end{array}\right]^{\mathrm{T}}$ $\mathrm{N}$, where $\Theta_{1}=2 \pi / 7 \mathrm{rad} /$ sample, $\Theta_{2}=2 \pi / 3 \mathrm{rad} /$ sample, and $\Theta_{3}=2 \pi / 17 \mathrm{rad} / \mathrm{sample}$. The open-loop system is given the initial conditions $q(0)=\left[\begin{array}{lll}-0.7 & 0.65 & -0.35\end{array}\right]^{\mathrm{T}} \mathrm{m}$, and $\dot{q}=\left[\begin{array}{ccc}0.5 & 0.1 & 0.3\end{array}\right]^{\mathrm{T}} \mathrm{m} / \mathrm{sec}$. The plant is simulated in open-loop for 100 seconds, and at $t=100 \mathrm{sec}$, RCAC is turned on with tuning parameters $n_{\mathrm{c}}=15, \eta_{0}=0.005, p_{c}=1, P_{0}=I$, and $K_{z u}$ as given above. The closed-loop response is shown in Figure 21. After convergence, the disturbance frequencies $\pi / 7 \mathrm{rad} /$ sample, $2 \pi / 3 \mathrm{rad} / \mathrm{sample}$ and $2 \pi / 17$ are attenuated as shown in Figure 10.
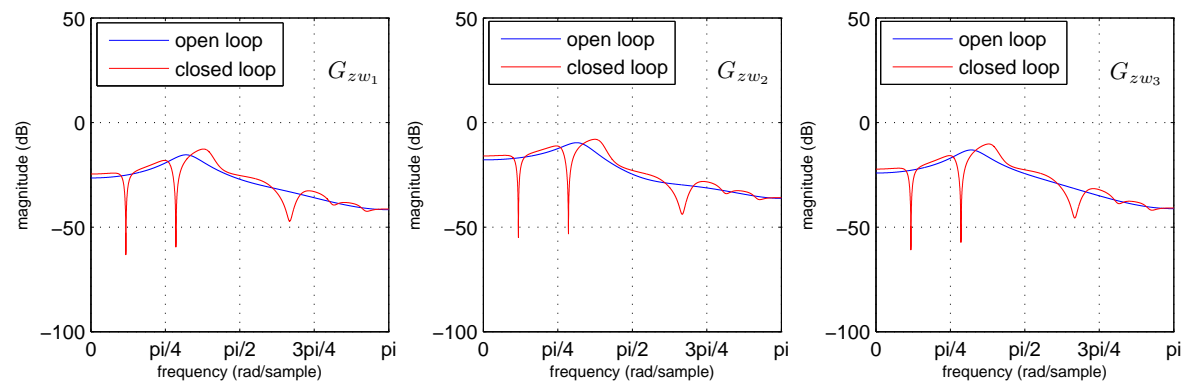

Figure 10. Example IV.7: 3DOF asymptotically stable uncertain structure. The bode plots show the attenuation at the disturbance frequencies $2 \pi / 7,2 \pi / 3$ and $2 \pi / 17 \mathrm{rad} /$ sample after controller convergence.

\section{IV.B. MIMO Examples}

In this section, we apply RCAC to MIMO flexible structures. The simulation results corresponding to the examples in this section are given in Appendix B.

Example IV.8 (Adaptive MIMO control of a 2DOF Lyapunov-stable lumped parameter structure). Consider a 2DOF lumped parameter structure with the masses $m_{1}=m_{2}=2 \mathrm{~kg}$; the spring constants $k_{1}=k_{3}=7 \mathrm{~kg} / \mathrm{sec}^{2}, k_{2}=0 \mathrm{~kg} / \mathrm{sec}^{2}$; and the damping coefficients $c_{1}=c_{3}=0 \mathrm{~kg} / \mathrm{sec}, c_{2}=3.5 \mathrm{~kg} / \mathrm{sec}$. 
With these parameters, for certain initial conditions, the two masses can oscillate at the same frequency with equal amplitudes and phases, so that the relative motion $\dot{q}_{1}-\dot{q}_{2}$ is equal to zero, and $c_{2}$ dissipates no energy. Therefore, $\bar{A}$ has two non-repeated eigenvalues on the imaginary axis, and the remaining eigenvalues lie in the open left-half plane, thus the structure is Lyapunov-stable. The continuous-time plant $T_{z u}(s)=$ $\bar{E}_{1}(s I-\bar{A})^{-1} \bar{B}$ is sampled at $2 \mathrm{~Hz}$ so that $T_{s}=0.5 \mathrm{sec} /$ sample. The sampled-data system $G_{z u}(z)$ has two nonminimum-phase channels, as shown in Figure 11. However, the transmission zeros -1 and -0.5559 of the MIMO transfer matrix $G_{z u}(z)$ are on or inside the unit circle.
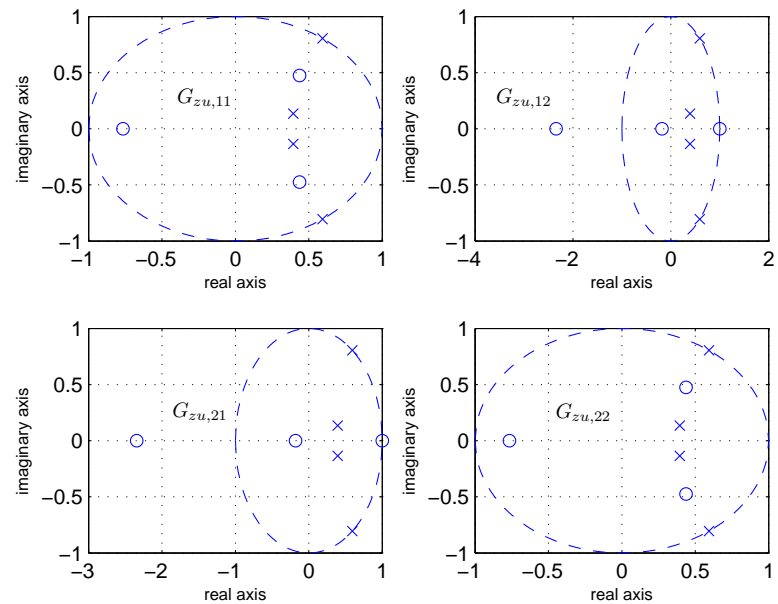

Figure 11. Example IV.8: Channel poles and zeros of $G_{z u}$.

The control objective is to keep $q_{1}$ and $q_{2}$ near zero in the presence of the disturbance forces $\bar{w}_{1}$ and $\bar{w}_{2}$, using the control forces $f_{1}$ and $f_{2}$. We assume the measurements of $q_{1}$ and $q_{2}$ are corrupted by mutually uncorrelated white noise sequences $v_{1}(k)$ and $v_{2}(k)$ having standard deviations 0.46 $\mathrm{m}$ and $0.43 \mathrm{~m}$ respectively. Therefore, we consider a MIMO disturbance rejection problem with $y=z=$ $\left[\begin{array}{ll}q_{1}\left(k T_{s}\right) & q_{2}\left(k T_{s}\right)\end{array}\right]^{\mathrm{T}}+\left[\begin{array}{ll}v_{1} & v_{2}\end{array}\right]^{\mathrm{T}}$. We consider the unknown sinusoidal disturbances $\bar{w}_{1}, \bar{w}_{2}$ with frequencies $\omega_{1}=0.2857 \mathrm{~Hz}, \omega_{2}=0.087 \mathrm{~Hz}$, that is, $w(k)=\left[\begin{array}{ll}w_{1}(k) & w_{2}(k)\end{array}\right]^{\mathrm{T}}=\left[\begin{array}{cc}15 \sin \Theta_{1} k & 10 \sin \Theta_{2} k\end{array}\right]^{\mathrm{T}}$ $\mathrm{N}$, where $\Theta_{1}=2 \pi / 7 \mathrm{rad} /$ sample and $\Theta_{2}=2 \pi / 23 \mathrm{rad} /$ sample. The open-loop system is given the initial conditions $q(0)=\left[\begin{array}{ll}-0.05 & 0.3\end{array}\right]^{\mathrm{T}} \mathrm{m}$ and $\dot{q}=\left[\begin{array}{ll}0.15 & -0.4\end{array}\right]^{\mathrm{T}} \mathrm{m} / \mathrm{sec}$. The plant is simulated in open-loop for 50 seconds, and at $t=50 \mathrm{sec}, \mathrm{RCAC}$ is turned on with tuning parameters $n_{\mathrm{c}}=15, \eta_{0}=0, P_{0}=I$, and $K_{z u}=H_{1}$. The masses oscillate until $t=50 \mathrm{sec}$, and then are brought back near zero as shown in Figure 22.

Example IV.9 (Adaptive MIMO control of a 4DOF asymptotically stable lumped parameter structure). Consider a 4 DOF lumped parameter structure with the masses $m_{1}=3 \mathrm{~kg}, m_{2}=4 \mathrm{~kg}, m_{3}=5 \mathrm{~kg}$, $m_{4}=6 \mathrm{~kg}$; the spring constants $k_{1}=7 \mathrm{~kg} / \mathrm{sec}^{2}, k_{2}=6 \mathrm{~kg} / \mathrm{sec}^{2}, k_{3}=7 \mathrm{~kg} / \mathrm{sec}^{2}, k_{4}=8 \mathrm{~kg} / \mathrm{sec}^{2}, k_{5}=6$ $\mathrm{kg} / \mathrm{sec}^{2}$; and the damping coefficients $c_{1}=1.5 \mathrm{~kg} / \mathrm{sec}, c_{2}=1.3 \mathrm{~kg} / \mathrm{sec}, c_{3}=1.9 \mathrm{~kg} / \mathrm{sec}, c_{4}=2 \mathrm{~kg} / \mathrm{sec}$, and $c_{5}=1.9 \mathrm{~kg} / \mathrm{sec}$. With these parameters, every eigenvalue of $\bar{A}$ lies in the open left-half plane, thus the structure is asymptotically stable. The continuous-time plant $T_{z u}(s)=\bar{E}_{1}(s I-\bar{A})^{-1} \bar{B}$ is sampled at $2 \mathrm{~Hz}$ so that $T_{s}=0.5 \mathrm{sec} /$ sample. The sampled-data system $G_{z u}(z)$ has three nonminimum-phase channels, as shown in Figure 12, and, the MIMO transfer matrix $G_{z u}(z)$ has a nonminimum-phase sampling zero near -4.03 .

The control objective is to keep $q_{3}$ and $q_{4}$ near zero in the presence of the disturbance forces $\bar{w}_{1}$, $\bar{w}_{2}$ and $\bar{w}_{4}$, using the control forces $f_{2}$ and $f_{4}$. We assume that the measurements of $q_{3}$ and $q_{4}$ are available, thus $y=z=\left[\begin{array}{ll}q_{3} & q_{4}\end{array}\right]^{\mathrm{T}}$. We consider the unknown sinusoidal disturbances $\bar{w}_{1}, \bar{w}_{2}$ and $\bar{w}_{4}$ with frequencies $\omega_{1}=0.8 \mathrm{~Hz}, \omega_{2}=0.069 \mathrm{~Hz}, \omega_{4}=0.1176 \mathrm{~Hz}$, that is, $w(k)=\left[\begin{array}{lll}w_{1}(k) & w_{2}(k) & w_{4}(k)\end{array}\right]^{\mathrm{T}}=$ 

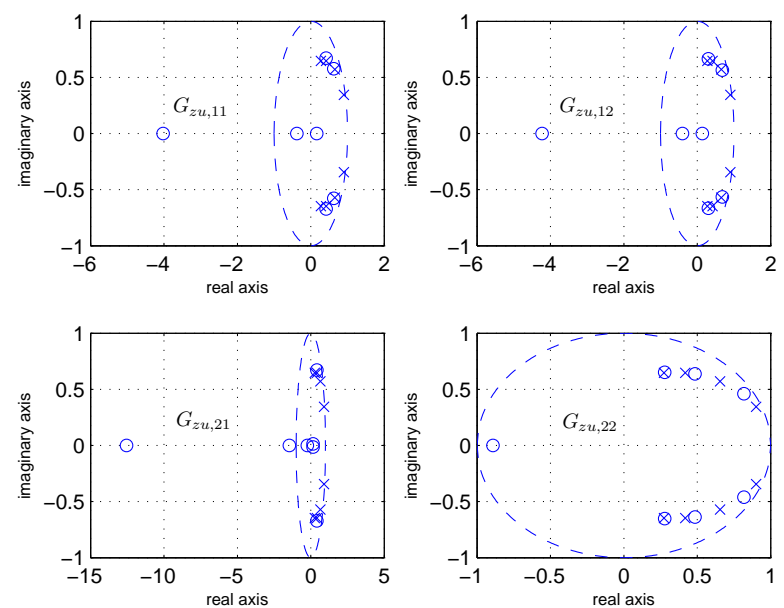

Figure 12. Example IV.9: Channel poles and zeros of $G_{z u}$.

$\left[\begin{array}{lll}20 \sin \Theta_{1} k & 20 \sin \Theta_{2} k & 20 \sin \Theta_{4} k\end{array}\right]^{\mathrm{T}} \mathrm{N}$, where $\Theta_{1}=4 \pi / 5 \mathrm{rad} / \mathrm{sample}, \Theta_{2}=2 \pi / 29 \mathrm{rad} / \mathrm{sample}$, and

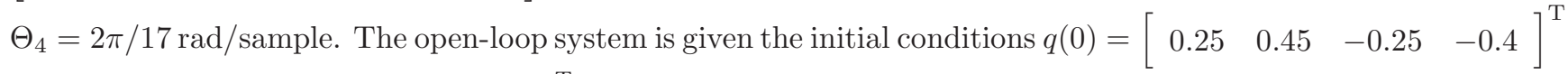
$\mathrm{m}$ and $\dot{q}=\left[\begin{array}{llll}0.05 & 0.3 & -0.2 & -0.05\end{array}\right]^{\mathrm{T}} \mathrm{m} / \mathrm{sec}$. The plant is simulated in open-loop for 50 seconds, and at $t=50 \mathrm{sec}, \mathrm{RCAC}$ is turned on with tuning parameters $n_{\mathrm{c}}=20, \eta_{0}=0.1, p_{c}=5, P_{0}=0.1 I$, and $K_{z u}=H_{1}$. The closed-loop response is shown in Figure 23.

\section{Conclusions}

In this paper we applied the latest developments in RCAC to the active vibration control problem. We demonstrated disturbance rejection in lightly damped structures with uncertain modal frequencies, damping, and mode shapes, uncertain disturbance spectra, sensor noise, and without the benefit of disturbance measurements, and sensor/actuator passivity due to colocation and velocity sensing, in both SISO and MIMO applications. We considered physical lumped parameter structure models which may exhibit nonminimumphase behavior due to sample and hold operations. In these cases, we demonstrated that satisfying a phase-matching condition is sufficient for asymptotic suppression of exogenous disturbance forces. 


\section{A. Simulation Results for Section IV.A}

In this appendix, we present simulation results corresponding to SISO numerical examples considered in Section IV.A. Each figure contains 8 subplots arranged in four rows and two columns. In each figure, first row shows the time history of the performance variable $z$ which may consist of displacements $q_{i}(t)$ or velocities $\dot{q}_{i}(t)$, and the control signal $u$, which may consist of control forces $f_{i}(t)$; second row shows the time traces of the controller gain vector $\Theta(k)$ and the spectral radius $\operatorname{spr}(\tilde{A})$ of the closed-loop state matrix; third row shows the pole-zero maps of the continuous-time plant $T_{z u}(s)$ and the corresponding sampled-data system $G_{z u}(z)$; finally, fourth row shows the phase mismatch $\Delta(\theta)$ and the bode magnitude plot of the controller transfer function after convergence, plotted for all $\theta \in[0, \pi] \mathrm{rad} / \mathrm{sample}$, where 0 corresponds to the DC-frequency and $\pi$ corresponds to the Nyquist frequency.
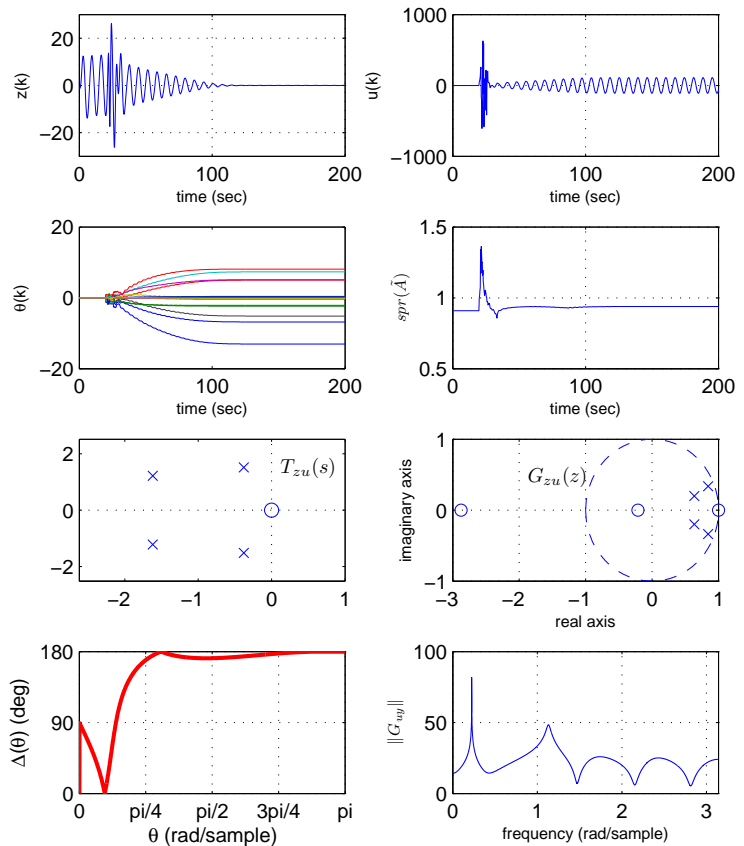

Figure 13. Example IV.1: 2DOF, asymptotically stable structure, sampled with $T_{s}=0.25 \mathrm{sec} / \mathrm{sample}$. The sampled-data system has a NMP sampling zero. The control objective is to keep $q_{2}(t)$ near zero in the presence of the disturbance force $\bar{w}_{2}(t)=100 \sin (2 \pi t / 7) \mathbf{N}$ using the control force $f_{1}$. RCAC is turned on at $t=25$ sec with the tuning parameters $n_{\mathrm{c}}=10, \eta_{0}=0.5, P_{0}=10 I, p_{c}=1$, and $K_{z u}=H_{1}=0.002$. With this choice of $K_{z u}$, the phase mismatch is smaller than $90 \mathrm{deg}$ at the disturbance frequency $\Theta_{1}=\pi / 14 \mathrm{rad} / \mathrm{sample}$. The controller gain vector $\Theta(k)$ converges, and $q_{2}$ converges to zero in about 70 seconds (280 time steps). RCAC converges to an internal model controller with high-gain at the disturbance frequency. After convergence, the spectral radius $\operatorname{spr}(\tilde{A})$ of the closed-loop system is 0.94 .

\section{B. Simulation Results for Section IV.B}

In this appendix, we present simulation results corresponding to the MIMO numerical examples considered in Section IV.B. Each figure contains 6 subplots arranged in three rows and two columns. In each figure, first row shows the time history of the performance variable $z$, which may consist of displacements $q_{i}(t)$ or velocities $\dot{q}_{i}(t)$, and the control signal $u$, which may consist of control forces $f_{i}(t)$; second row shows the time traces of the controller gain vector $\Theta(k)$ and the spectral radius $\operatorname{spr}(\tilde{A})$ of the closed-loop state matrix; third row shows the poles and transmission zeros of the continuous-time MIMO transfer function $T_{z u}(s)$ and the corresponding sampled-data system $G_{z u}(z)$. 

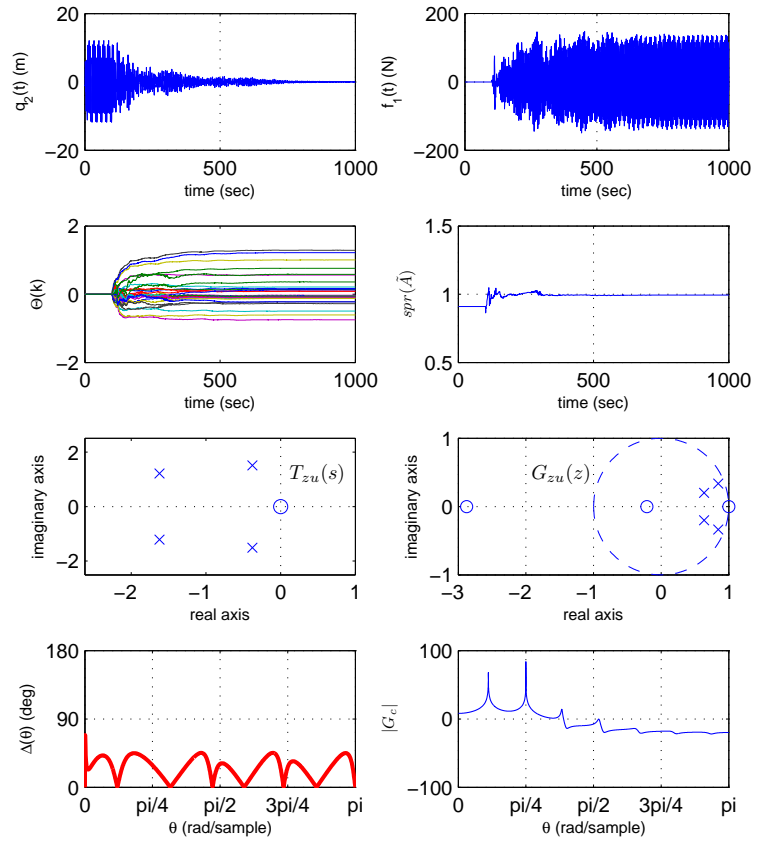

Figure 14. Example IV.1: 2DOF, asymptotically stable structure sampled with $T_{s}=0.25$ sec/sample. The sampled-data system has a NMP sampling zero. The control objective is to keep $q_{2}(t)$ near zero in the presence of the disturbance forces $\bar{w}_{1}(t)=100 \sin (2 \pi t / 2) \mathbf{N}$ and $\bar{w}_{2}(t)=10 \sin \left(2 \pi \frac{2}{9} t\right) \mathbf{N}$ using the control force $f_{1}$. RCAC is turned on at $t=100$ sec with the tuning parameters $n_{\mathrm{c}}=15, \eta_{0}=0.1, P_{0}=0.1 I$, and $p_{c}=5 . \quad K_{z u}$ is constructed so that $\Delta(\theta)<45$ deg for all $\theta \in[0, \pi] \mathrm{rad} / \mathrm{sample}$. The controller gain vector $\Theta(k)$ converges, and $q_{2}$ converges to zero in about 400 seconds (2000 time steps). The performance variable does not exceed the open-loop during the transient period. After convergence, the spectral radius $\operatorname{spr}(\tilde{A})$ of the closed-loop system is 0.99 .
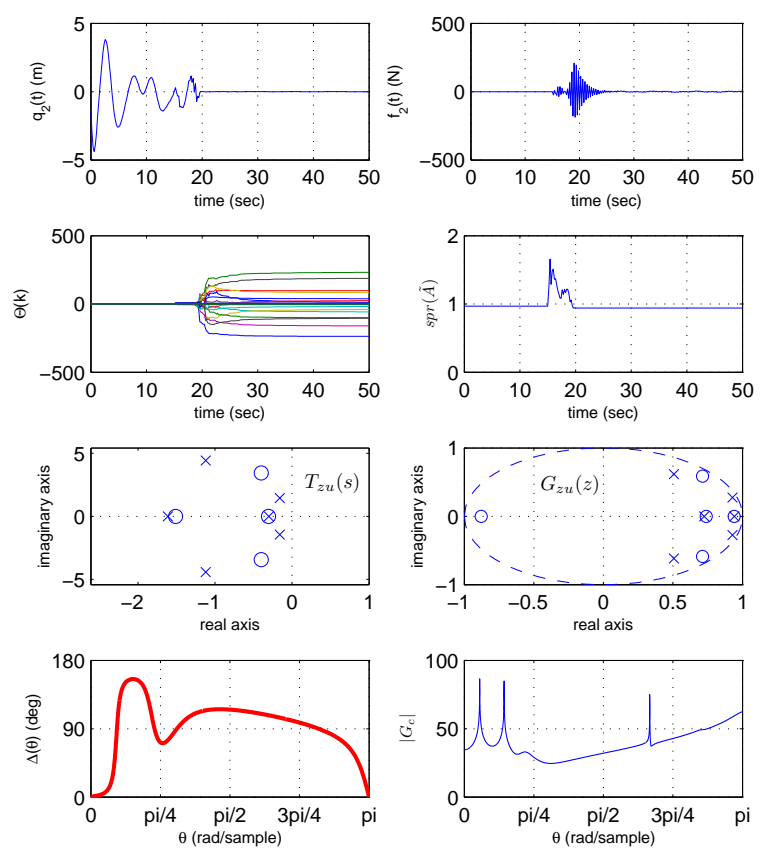

Figure 15. Example IV.2: 3DOF, asymptotically stable structure, sampled with $T_{s}=0.2$ sec/sample. The control objective is to keep $q_{2}(t)$ near zero in the presence of the disturbance forces $\bar{w}_{1}(t)=10 \sin \left(2 \pi \frac{5}{14} t\right) \mathbf{N}, \bar{w}_{2}(t)=2 \sin \left(2 \pi \frac{5}{36} t\right)$ $\mathbf{N}$ and $\bar{w}_{3}(t)=15 \sin \left(2 \pi \frac{5}{3} t\right) \mathbf{N}$, using the control force $f_{2}$. RCAC is turned on at $t=15$ sec with the tuning parameters $n_{\mathrm{c}}=15, \eta_{0}=0, P_{0}=10^{10} I$, and $K_{z u}=H_{1}$. The controller gain vector $\Theta(k)$ converges, and $q_{2}$ converges to zero in about 5 seconds (25 time steps). The performance variable does not exceed the open-loop during the transient period. After convergence, the spectral radius $\operatorname{spr}(\tilde{A})$ of the closed-loop system is 0.94 . 

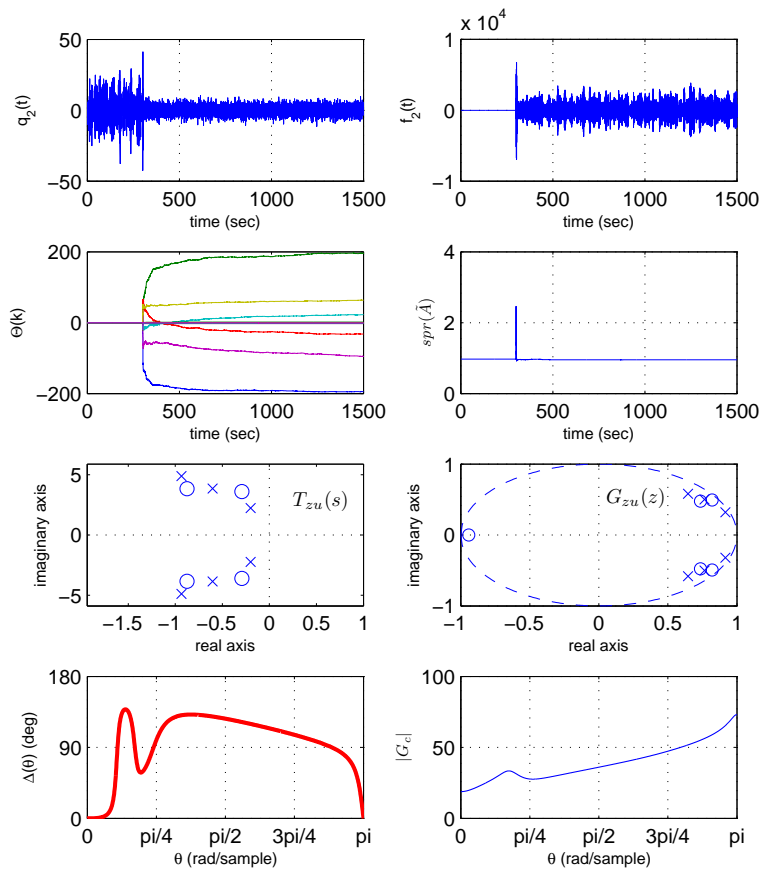

Figure 16. Example IV.3: 3DOF, asymptotically stable structure, sampled with $T_{s}=0.15$ sec/sample. The control objective is to keep $q_{2}(t)$ near zero in the presence of the bandlimited white disturbance forces $\bar{w}_{1}(k), \bar{w}_{2}(k)$ and $\bar{w}_{3}(k)$ with standard deviations $\sigma_{w_{1}}=157.29 \mathrm{~N}, \sigma_{w_{2}}=99.81 \mathrm{~N}, \sigma_{w_{3}}=153.97 \mathrm{~N}$, using the control force $f_{2}$. The disturbances are uncorrelated with each other, and the correlation time for each disturbance is 0.03 sec. RCAC is turned on at $t=40$ sec with the tuning parameters $n_{c}=6, \eta_{0}=0, P_{0}=100 I$, and $K_{z u}=H_{1}$. The performance variable $q_{2}$ is brought below the open-loop level, and at $t=1500 \mathrm{sec}$, the spectral radius $\operatorname{spr}(\tilde{A})$ of the closed-loop system is 0.95 .
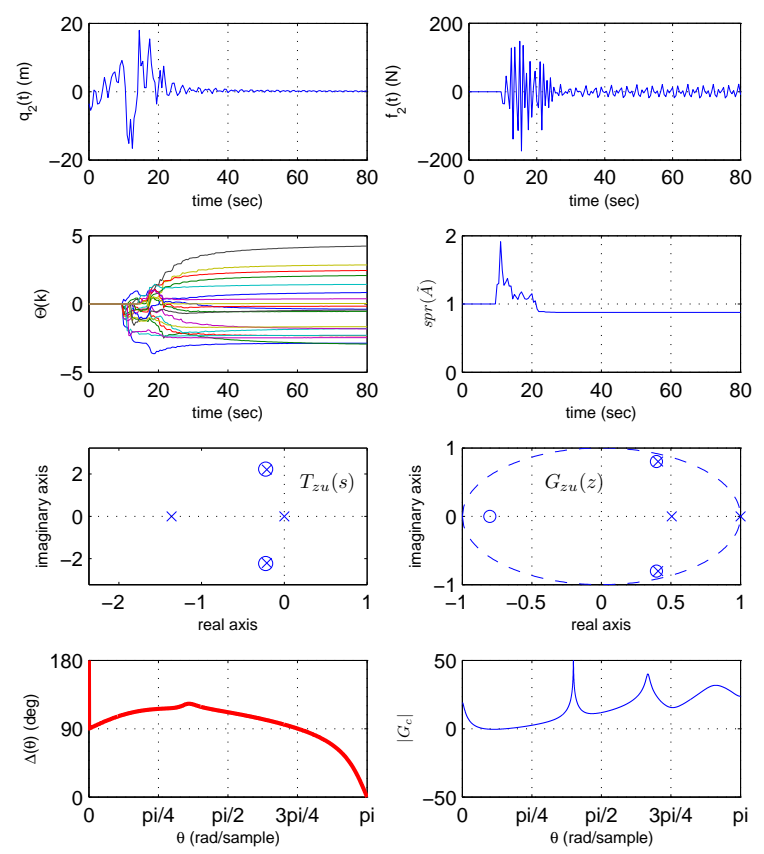

Figure 17. Example IV.4: 2DOF, semistable structure, sampled with $T_{s}=0.5$ sec/sample. The control objective is to keep $z=q_{2}(t)$ near zero in the presence of the disturbance forces $\bar{w}_{1}(t)=100 \sin \left(2 \pi \frac{2}{3} t\right) \mathbf{N}$ and $\bar{w}_{2}(t)=1+10 \sin \left(2 \pi \frac{2}{5} t\right) \mathbf{N}$, using the control force $f_{2}$. Note that $\bar{w}_{2}$ has a DC component that excites the damped rigid-body mode. RCAC is turned on at $t=10 \mathrm{sec}$ with the tuning parameters $n_{\mathrm{c}}=10, \eta_{0}=0, P_{0}=I$, and $K_{z u}=H_{1}$. The controller gain vector $\Theta(k)$ converges, and $q_{2}$ converges to zero in about 20 seconds (40 time steps). After convergence, the spectral radius $\operatorname{spr}(\tilde{A})$ of the closed-loop system is 0.88 . 

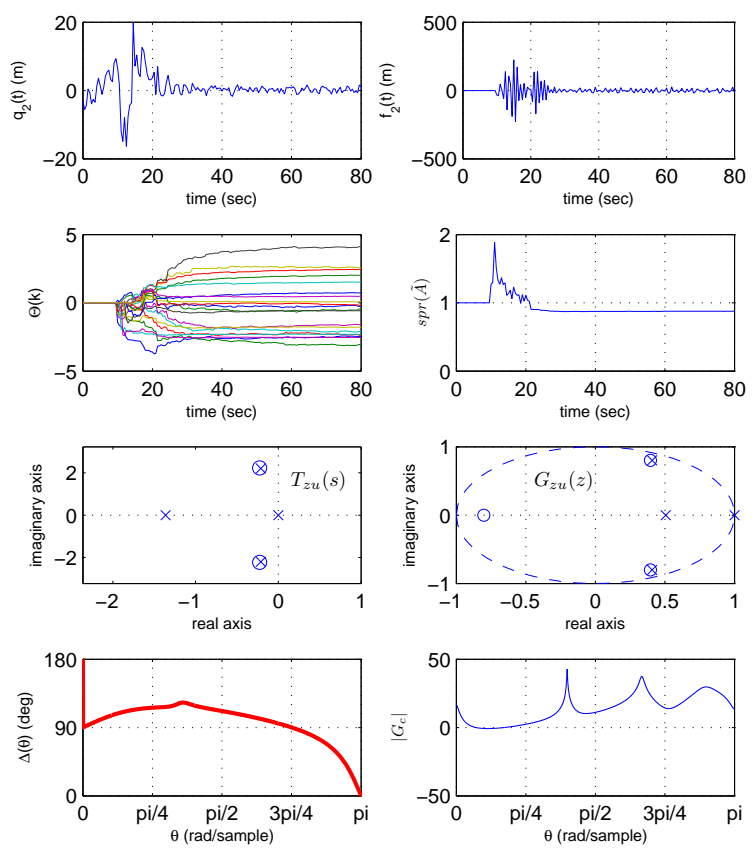

Figure 18. Example IV.4: 2DOF, semistable structure, sampled with $T_{s}=0.5$ sec/sample. The control objective is the same as in Figure 17, but now the measurements are corrupted by a gaussian white-noise with standard deviation 0.44 m. RCAC is turned on at $t=10$ sec with the same tuning parameters as in Figure 17 . The performance $q_{2}$ is driven near zero in about 20 seconds (40 time steps). The performance does not fully converge to zero due to the presence of measurement noise. The spectral radius $\operatorname{spr}(\tilde{A})$ of the closed-loop system is 0.88 at $t=80$ sec.
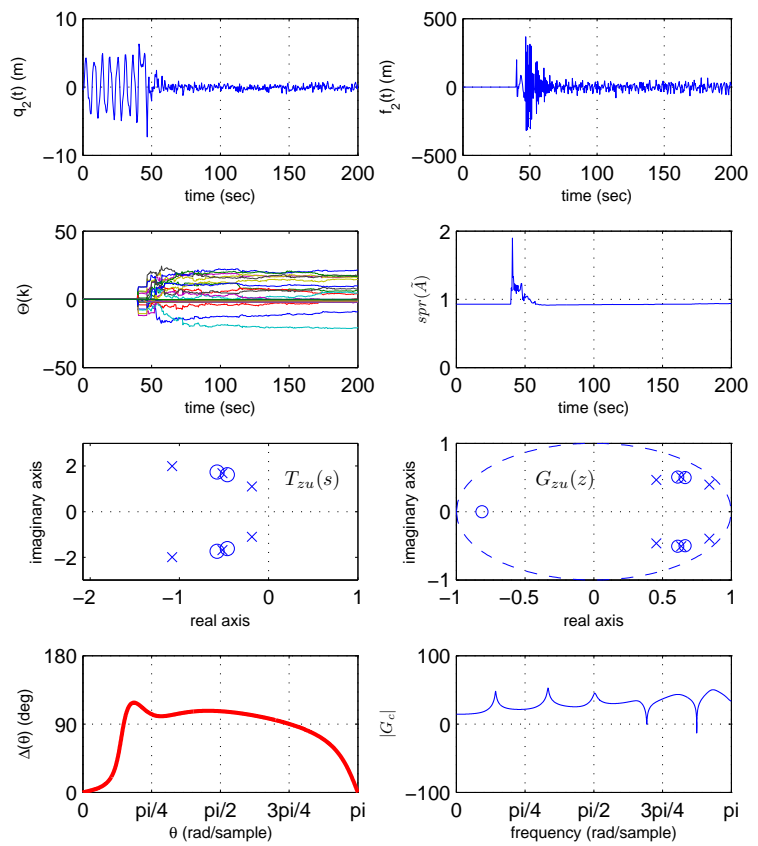

Figure 19. Example IV.5: 3DOF, asymptotically stable structure, sampled with $T_{s}=0.4$ sec/sample. The control objective is to keep $q_{2}$ near zero in the presence of the disturbance forces $\bar{w}_{1}(t)=80 \sin (2 \pi 0.625 t) \mathbf{N}, \bar{w}_{2}(t)=15 \sin (2 \pi 0.1786 t)$ and $\bar{w}_{3}(t)=70 \sin (2 \pi 0.4167 t)$, using the control force $f_{2}$. Furthermore, the measurements are corrupted by a gaussian white-noise with standard deviation $0.24 \mathrm{~m}$. RCAC is turned on at $t=40$ sec with the tuning parameters $n_{\mathrm{c}}=15, \eta_{0}=0$, $P_{0}=10^{3} \mathrm{I}$, and $K_{z u}=H_{1}=0.0165$. The controller gain vector $\Theta(k)$ converges, and $q_{2}$ is driven near zero in about 20 seconds (50 time steps). The performance does not fully converge to zero due to the presence of measurement noise. The spectral radius $\operatorname{spr}(\tilde{A})$ of the closed-loop system is 0.94 at $t=200$ sec. 

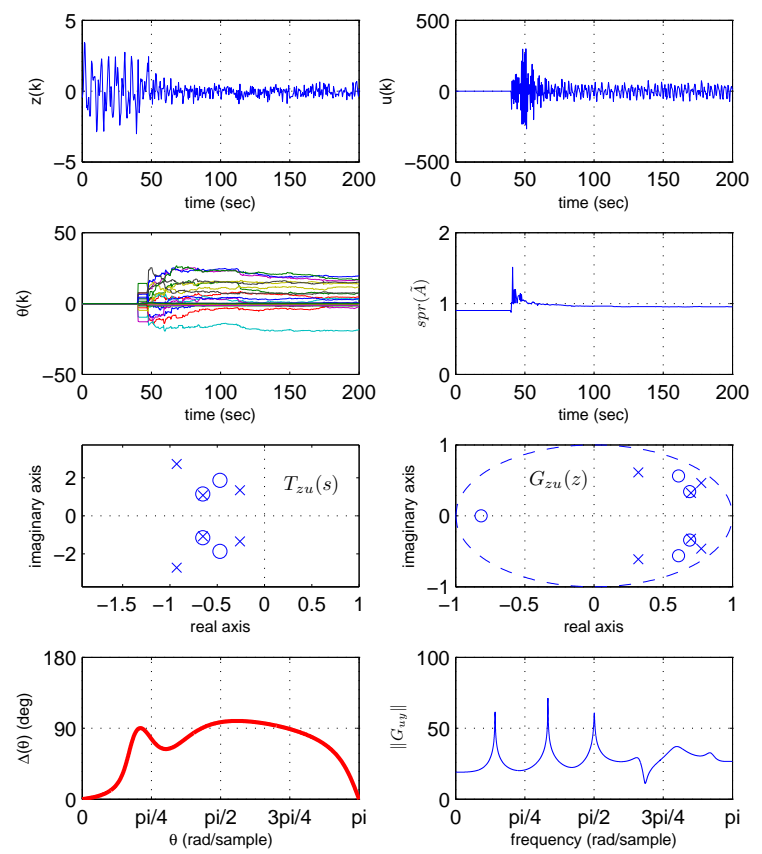

Figure 20. Example IV.6: 3DOF, asymptotically stable structure, sampled with $T_{s}=0.4$ sec/sample. The control objective is the same as in Figure 19, but now the mass, spring and damping matrices have uncertain components having equal norms to the corresponding known components. Furthermore, because of the uncertainties, the mass matrix $\tilde{M}$ is not diagonal. RCAC is turned on at $t=40$ sec with the same tuning parameters as in Figure 19, thus, $K_{z u}=K_{1}=0.0165$ is not equal to the first Markov parameter $H_{1}=0.0133$ of the actual uncertain system. The performance $q_{2}$ is driven near zero in about 30 seconds (75 time steps). The performance does not fully converge to zero due to the presence of measurement noise. The spectral radius $\operatorname{spr}(\tilde{A})$ of the closed-loop system is 0.95 at $t=200$ sec.

\section{References}

${ }^{1}$ M. J. Balas, "Trends in large space structures control theory: Fondest hopes, wildest dreams," IEEE Trans. Automat. Contr., vol. AC-27, pp. 522-535, 1982.

${ }^{2}$ S. M. Kuo and D. R. Morgan, Active Noise Control Systems. New York: Wiley-Interscience, 1996.

${ }^{3}$ P. A. Nelson and S. J. Elliott, Active Control of Sound. London: Academic, 1992.

${ }^{4}$ C. Y. Lai and W. H. Liao, "Vibration Control of a Suspension System via a Magnetorheological Fluid Damper," J. of Vibr. and Contr., vol.8, no.4, pp.527-547, April 2002.

${ }^{5}$ S. J. Dyke, B. F. Spencer Jr., M. K. Sain, and J. D. Carlson, "Modeling and Control of magnetorheological dampers for seismic response reduction", Smart Mater. and Struct., vol. 5, no.5, pp. 565-575, 1996.

${ }^{6}$ M. A. Karkoub and M. Zribi, "Active/semi-active suspension control using magnetorheological actuators", Int. J. of Sys. Sci., vol. 37 , no. 1 , pp. 35-44, 2006.

${ }^{7}$ J. Hong and D. S. Bernstein, "Bode Integral Constraints, Colocation, and Spillover in Active Noise and Vibration Control," IEEE Trans. Contr. Sys. Tech., vol. 6, pp. 111-120, 1998.

${ }^{8}$ C. R. Fuller and A. H. von Flotow, "Active Control of Sound and Vibration", IEEE Contr. Sys., vol. 15, no. 6, pp. 9-19, Dec 1995.

${ }^{9}$ I. R. Petersen and A. Lanzon, "Feedback Control of Negative-Imaginary Systems", IEEE Contr. Sys., vol. 30, no. 5, pp. 54-72, Oct 2010.

${ }^{10}$ D. Simon, Optimal State Estimation, 1st Edition, Wiley Publishing, 2006.

${ }^{11}$ CT. Chen, Linear Systems Theory and Design, 3rd Edition, Oxford University Press, 1998.

${ }^{12}$ D. S. Bernstein, Matrix Mathematics, $2^{\text {nd }}$ Edition, Princeton University Press, 2009.

${ }^{13}$ K. J. Astrom and B. Wittenmark, Adaptive Control, 2nd ed., Addison-Wesley, 1995.

${ }^{14}$ G. C. Goodwin and K. S. Sin, Adaptive Filtering, Prediction and Control, Prentice Hall, 1984.

${ }^{15}$ D. S. Bernstein and S. P. Bhat, "Lyapunov Stability, Semistability, and Asymptotic Stability of Matrix Second-Order Systems," ASME J. Mechanical Design, Vol. 117, Issue B, pp. 145-153, 1995.

${ }^{16}$ R. Venugopal and D. S. Bernstein. "Adaptive Disturbance Rejection Using ARMARKOV System Representations," IEEE Trans. Contr. Sys. Tech., Vol. 8, pp. 257-269, 2000.

${ }^{17}$ J. B. Hoagg, M. A. Santillo, and D. S. Bernstein, "Discrete-Time Adaptive Command Following and Disturbance Rejection for Minimum Phase Systems with Unknown Exogenous Dynamics," IEEE Trans. Autom. Contr., Vol. 53, pp. 912-928, 2008. 

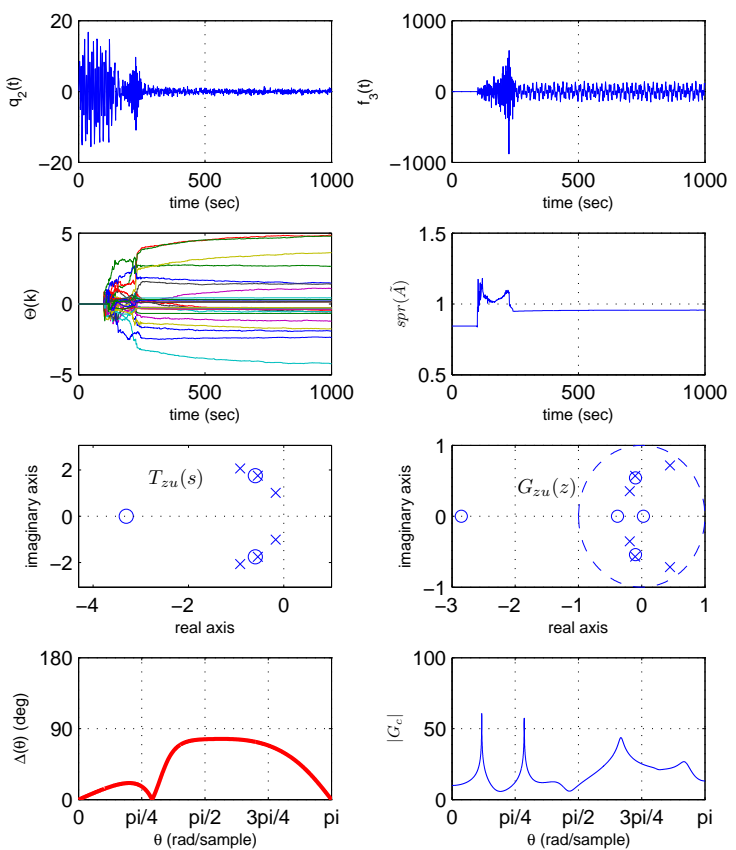

Figure 21. Example IV.7: 3DOF, asymptotically stable structure, sampled with $T_{s}=1$ sec/sample. The sampleddata system has a NMP sampling zero. The plant parameters are assumed to be completely unknown, and $K_{z u}$ is constructed using a constrained least squares method that fits the frequency response estimates of $G_{z u}$ with an FIR plant. The frequency response estimates are obtained with frequency domain system identification using a white-noise input sequence. The control objective is to keep $q_{2}$ near zero in the presence of the disturbance forces $\bar{w}_{1}(t)=75 \sin (2 \pi t / 7)$ $\mathbf{N}, \bar{w}_{2}(t)=30 \sin (2 \pi t / 3) \mathbf{N}$ and $\bar{w}_{3}(t)=70 \sin (2 \pi t / 17) \mathbf{N}$, using the control force $f_{3}$. Furthermore, the measurements are corrupted by a gaussian white-noise with standard deviation $0.32 \mathrm{~m}$. RCAC is turned on at $t=100$ sec with the tuning parameters $n_{\mathrm{c}}=15, \eta_{0}=0.005, p_{c}=1$ and $P_{0}=I$. The performance variable $q_{2}$ converges near zero in about 100 seconds (100 time steps), and the transient performance does not exceed the open-loop performance. The $\operatorname{spectral} \operatorname{radius~spr}(\tilde{A})$ of the closed-loop system is 0.96 at $t=1000$ sec. 

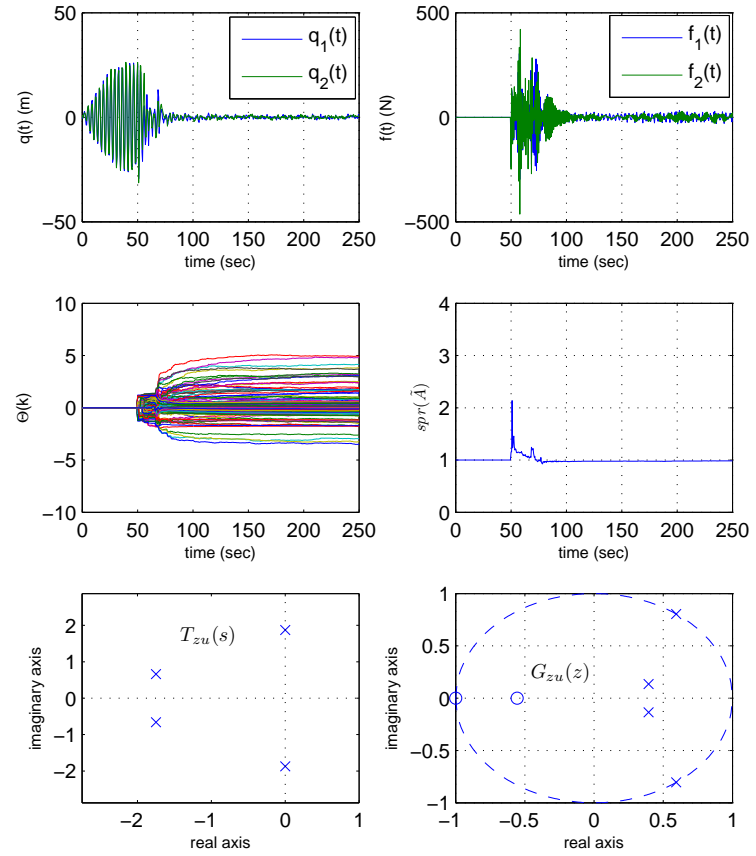

Figure 22. Example IV.8: 2DOF, Lyapunov-stable structure, sampled with $T_{s}=0.5 \mathrm{sec} / \mathrm{sample}$. The sampled-data system has a sampling zero at -1 . The control objective is to keep $q_{1}$ and $q_{2}$ near zero in the presence of the disturbance forces $\bar{w}_{1}=15 \sin (2 \pi 0.2857 t), \bar{w}_{2}=10 \sin (2 \pi 0.087 t)$ using the control forces $f_{1}$ and $f_{2}$. The measurements of $q_{1}$ and $q_{2}$ are corrupted by mutually uncorrelated white noise sequences with standard deviations $0.46 \mathrm{~m}$ and $0.43 \mathrm{~m}$ respectively. RCAC is turned on at $t=50$ sec with $n_{\mathrm{c}}=15, \eta_{0}=0, P_{0}=I$, and $K_{z u}=H_{1}$. The displacements $q_{1}$ and $q_{2}$ are driven near zero in about 50 seconds (100 time steps), and the transient performance does not exceed the open-loop performance. The spectral radius $\operatorname{spr}(\tilde{A})$ of the closed-loop system is 0.98 at $t=250 \mathrm{sec}$.
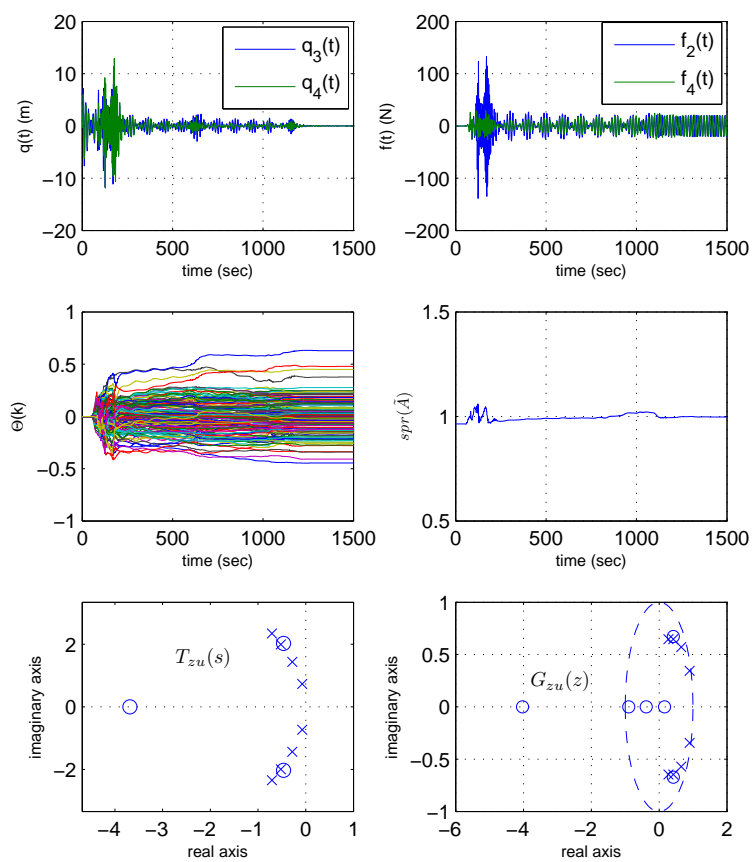

Figure 23. Example IV.9: 2DOF, Lyapunov-stable structure, sampled with $T_{s}=0.5 \mathrm{sec} / \mathrm{sample}$. The sampled-data system has a nonminimum-phase sampling zero near -4.03 . The control objective is to keep $q_{3}$ and $q_{4}$ near zero in the presence of the disturbance forces $\bar{w}_{1}=20 \sin (2 \pi 0.8 t) \mathbf{N}, \bar{w}_{2}=20 \sin (2 \pi 0.069 t) \mathbf{N}$, and $\bar{w}_{4}=20 \sin (2 \pi 0.1176 t)$, using the control forces $f_{2}$ and $f_{4}$. RCAC is turned on at $t=50$ sec with $n_{\mathrm{c}}=20, \eta_{0}=0.1, p_{c}=5, P_{0}=0.1 I$, and $K_{z u}=H_{1}$. The displacements $q_{3}$ and $q_{4}$ are driven near zero in about 1000 seconds (2000 time steps. The spectral $\operatorname{radius} \operatorname{spr}(\tilde{A})$ of the closed-loop system is 0.997 at $t=250$ sec. 
${ }^{18}$ M. A. Santillo and D. S. Bernstein, "Adaptive Control Based on Retrospective Cost Optimization," AIAA J. Guid. Contr. Dyn., Vol. 33, pp. 289-304, 2010.

${ }^{19}$ A. M. D'Amato, E. D. Sumer, and D. S. Bernstein, "Retrospective Cost Adaptive Control for Systems with Unknown Nonminimum-Phase Zeros," AIAA Guid. Nav. Contr. Conf., Portland, OR, August 2011, AIAA-2011-6203.

${ }^{20}$ A. M. D'Amato, E. D. Sumer, and D. S. Bernstein, "Frequency-Domain Stability Analysis of Retrospective-Cost Adaptive Control for Systems with Unknown Nonminimum-Phase Zeros," Proc. Conf. Dec. Contr., pp. 1098-1103, Orlando, FL, December 2011.

${ }^{21}$ E. D. Sumer, A. M. D'Amato, A. M. Morozov, J. B. Hoagg, and D. S. Bernstein, "Robustness of Retrospective Cost Adaptive Control to Markov-Parameter Uncertainty," Proc. Conf. Dec. Contr., pp. 6085-6090, Orlando, FL, December 2011.

${ }^{22}$ A. M. D'Amato, E. D. Sumer, K. S. Mitchell, A. V. Morozov, J. B. Hoagg, and D. S. Bernstein, "Adaptive Output Feedback Control of the NASA GTM Model with Unknown Nonminimum-Phase Zeros," AIAA Guid. Nav. Contr. Conf., Portland, OR, August 2011, AIAA-2011-6204.

${ }^{23}$ E. D. Sumer, M. H. Holzel, A. M. D'Amato, and D. S. Bernstein, "FIR-Based Phase Matching for Robust Retrospective-Cost Adaptive Control," Proc. Amer. Contr. Conf., pp. 2707-2712, Montreal, Canada, June 2012.

${ }^{24}$ E. D. Sumer, D. S. Bernstein, "Retrospective Cost Adaptive Control with Error-Dependent Regularization for MIMO Systems with Uncertain Nonminimum-Phase Transmission Zeros," AIAA Guid. Nav. Contr. Conf., Minneapolis, MN, 2012. 